\title{
Dynamic Effective Properties of Lead-Reinforced Epoxy Resin Composites with Differential Method
}

\author{
Haitao Zhu and Yongping Wan \\ School of Aerospace Engineering and Applied Mechanics, Tongji University, No. 1239 Siping Road, Shanghai 200092, China \\ Correspondence should be addressed to Yongping Wan; wanyp@tongji.edu.cn
}

Received 2 November 2021; Revised 19 January 2022; Accepted 22 January 2022; Published 12 February 2022

Academic Editor: Claudio Pettinari

Copyright (c) 2022 Haitao Zhu and Yongping Wan. This is an open access article distributed under the Creative Commons Attribution License, which permits unrestricted use, distribution, and reproduction in any medium, provided the original work is properly cited.

\begin{abstract}
In this paper, the quasi-P wave propagation in epoxy/lead composites is studied by the differential method with polarization stress and polarization momentum. The corresponding differential equations are numerically solved by the fourth-order Runge-Kutta method, and the dynamic effective modulus and density are obtained. Correctness of the results obtained by the differential method is verified by comparing with the results obtained by the self-consistent method as well as experiments. Results show that the dynamic effective modulus $\mathbf{L}_{1133}$ and $\mathbf{L}_{3333}$ increase with the increase of aspect ratio $\delta$, whereas $\mathbf{L}_{1111}$ and $\mathbf{L}_{1122}$ decrease with the increase of $\delta$. The phase velocity ratio of the quasi-P wave propagation in the composites tends to 1 when the dimensionless wave number is large, and the dynamic effective density and modulus obtained are not isotropic when $\delta \neq 1$. It is believed that the differential method can be a choice for solving dynamic effective properties of heterogeneous composites.
\end{abstract}

\section{Introduction}

In daily life, epoxy/lead composites have attracted more and more attention and been applied in many fields [1-3] since epoxy resins have the advantages of being green and degradable. The actual working environment of these composites is complex and changeable, especially in the case of nondestructive testing carried out on the composites with wave propagation. When damage degree of the composites is appraised during this working process, we need to know the dynamic effective properties of the composites.

The study of dynamic effective properties of composites mainly includes two parts: experiments and theoretical solutions. In the experimental aspect, Tauchert and Guzelsu tested the propagation of $\mathrm{P}$-wave and shear wave in spherical particle-reinforced boron-epoxy composite [4]. Kinra tested the propagation of $\mathrm{P}$-wave in the spherical and randomly distributed glass spheres/epoxy, lead spheres/epoxy, and steel spheres/PMMA composites, respectively [5]. Wang and Yuan tested the propagation of higher-order Lamb waves in composite plates [6].
In terms of theory, the dynamic constitutive relationship is constructed by ensemble average analysis with periodicity assumption by considering periodic composites and Bloch wave. Willis used Fourier series expansion and ensemble average to derive Green's function and the analytical dynamic effective modulus and density for one-dimensional periodic elastic composite [7]. Nemat-Nasser used the dynamic equivalent inclusion method to construct the dynamic effective constitutive relation in the case of periodic layered elastic composite by introducing eigenstress and eigenvelocity [8]. Srivastava and Nemat-Nasser derived the dynamic constitutive relation for the case of cuboid inclusion embedded in the cube matrix of pure elastic unit cell by introducing the eigenstrain and eigenmomentum with the dynamic equivalent inclusion method [9]. Sigalas and Soukoulis analyzed and studied the propagation of elastic waves in periodic layered composite by the transfer matrix method [10]. Hou et al. analyzed and studied the transmission spectra of elastic wave propagation in two-dimensional lead-epoxy composite [11, 12]. Sigalas and Economou used the wave function expansion method to 
analyze and calculate the bandgap characteristics of elastic waves propagating in periodic phononic crystals [13]. Yan and Wang calculated the band gap structure and fluid-solid coupling system of one-dimensional periodic gold-epoxy composites by wavelet analysis [14]. García-Pablos et al. firstly analyzed and studied the frequency band structure of elastic wave propagation in composites by the using finitedifference time-domain method, and verified the calculated results by comparing with experimental results [15]. Kafesaki and Economou used the multiple scattering method to analyze and calculate the frequency band of elastic wave propagation in three-dimensional periodic composites [16]. Kohn et al. used the variational method to analyze and study the dispersion relations of harmonic wave propagation in layered periodic elastic composites [17]. Hussein analyzed the calculation of phononic crystal, photonic crystal, and electronic band structure, respectively, by means of the reduced Bloch mode expansion [18].

Another theoretical method is based on the representative volume element (RVE) with the homogenization method, where the inclusion is randomly distributed in the composites. Tsinopoulos et al. solved the longitudinal wave and shear wave propagation in the random particle-reinforced composite with the iterative effective medium approximation method [19]. Verbis et al. used this method to analyze and solve the $\mathrm{P}$ wave, $\mathrm{SH}$ wave, and SV wave propagation in two-dimensional composite [20]. Kanaun and Levin used effective medium method to analyze and solve the case of axial shear wave propagation in infinite cylinder fiber composite [21]. Kim et al. studied the dynamic effective properties of longitudinal and shear wave propagation in spherical particle-reinforced two-phased composites with asymptotic expansion of the potential function and the self-consistent method [22]. Sabina et al. generalized the static self-consistent method to the dynamic situation by introducing polarization stress and polarization momentum and analyzed the dynamic effective properties of spherical particle-reinforced two-phased composites with $\mathrm{P}$ wave and $S$ wave propagated in the composites [23] and quasi-longitudinal wave and transverse wave in spherical particlereinforced composites and hole problems [24, 25]. Bussink et al. analyzed and studied the dynamic effective properties of longitudinal wave and shear wave propagation in twodimensional lead-reinforced epoxy [26].

On the other hand, the differential method is usually used to solve the static problems. Studies have proved the efficiency of the differential method [27-29]. In this paper, we study the possibility of analyzing the dynamic effective properties of two-phase particulate reinforced composites using the differential method. The quasi-P wave propagation in epoxy/lead composites is considered. By introducing the polarization stress and polarization momentum proposed by Willis [30], the differential method is generalized to analyze and solve the dynamic effective properties. The composite is assumed to be uniform macroscopically, while on the microscopic level, the inhomogeneities, with the same shape, size, and orientation as well as material properties, can be translationally but not rotationally scattered in the epoxy. The work of this paper is as follows. In Section 2, the materials and basic equations are introduced. Then, by introducing the scattering problem of approximate solution, the differential method is generalized to dynamic situation and differential equations are given, where the specific forms of differential equations are shown in appendix B. In Section 3 , by the use of the fourth-order Runge-Kutta method, the differential equations are numerically solved for different aspect ratios. Phase velocity ratios obtained by the differential method are compared to the self-consistent method and experimental results for verification. Also, dynamic effective modulus and density obtained by the differential method are compared to the results obtained by the selfconsistent method. In Section 4, conclusions of this paper are given.

\section{Materials and Methods}

For the sake of simplicity, quasi-P wave propagating in a two-phased linear elastic composite material is considered. The wave propagates along the negative direction parallel to the axis $\mathbf{x}_{3}$, and the material's principal axes of properties are fixed and only translational. The composite structure is shown in Figure 1, where the spheroid is inclusion and outside the spheroid is matrix.

Spheroidal inclusions satisfy the following equation:

$$
\Omega=\left\{\mathbf{x}: \mathbf{x}_{1}^{2}+\mathbf{x}_{2}^{2}+\frac{\mathbf{x}_{3}^{2}}{\delta^{2}}<a^{2}\right\},
$$

where $\delta$ is the aspect ratio and $a$ is the radius.

2.1. Basic Equations. Generally, the motion equation of the linear elastic material is

$$
\operatorname{div} \boldsymbol{\sigma}=\dot{\mathbf{p}}
$$

The constitutive equation is

$$
\begin{aligned}
& \boldsymbol{\sigma}=\mathbf{L} \mathbf{e}, \\
& \mathbf{p}=\boldsymbol{\rho} \mathbf{v},
\end{aligned}
$$

where $\boldsymbol{\sigma}$ denotes stress, $\mathbf{L}$ denotes modulus, e denotes strain, $\mathbf{p}$ denotes momentum, $\boldsymbol{\rho}$ denotes density, $\mathbf{v}$ denotes velocity, and the dot above the momentum $\mathbf{p}$ denotes derivative with respect to time.

The relation between velocity $\mathbf{v}$ and displacement $\mathbf{u}$ is

$$
\begin{aligned}
e_{i j} & =\frac{1}{2}\left(u_{i, j}+u_{j, i}\right), \\
v_{i} & =\dot{u}_{i} .
\end{aligned}
$$

Due to the linearity of equations (2) and (3), considering the ensemble average of equation (2), we have

$$
\operatorname{div}\langle\boldsymbol{\sigma}\rangle=\langle\dot{\mathbf{p}}\rangle,
$$

where $\langle\cdot\rangle$ represents ensemble average. Considering the two-phased composite material, where the modulus of the matrix is $\mathbf{L}_{2}$, density is $\boldsymbol{\rho}_{2}$, strain is $\mathbf{e}_{2}$, volume fraction of 


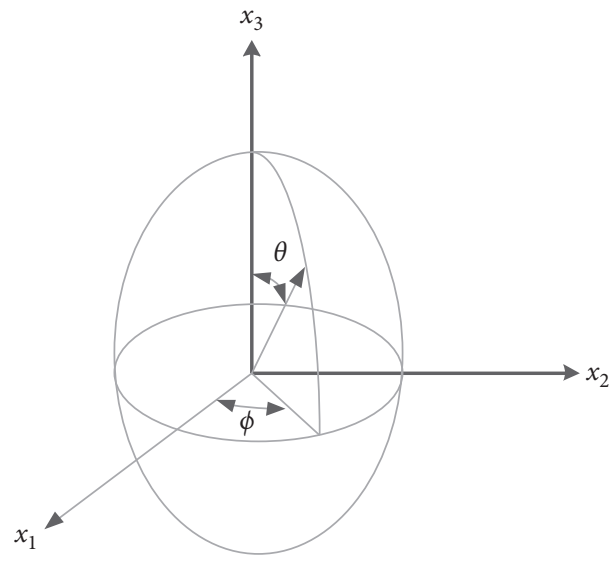

Figure 1: Illustration of inclusions.

matrix is $f_{2}$, the modulus of inclusion is $\mathbf{L}_{1}$, density is $\boldsymbol{\rho}_{1}$, strain is $\mathbf{e}_{1}$, and volume fraction of inclusion is $f_{1}$, we have

$$
\begin{aligned}
\langle\mathbf{e}\rangle & =f_{1}\langle\mathbf{e}\rangle_{1}+f_{2}\langle\mathbf{e}\rangle_{2}, \\
\langle\mathbf{v}\rangle & =f_{1}\langle\mathbf{v}\rangle_{1}+f_{2}\langle\mathbf{v}\rangle_{2}, \\
\langle\boldsymbol{\sigma}\rangle & =f_{1}\langle\boldsymbol{\sigma}\rangle_{1}+f_{2}\langle\boldsymbol{\sigma}\rangle_{2} \\
& =f_{1} \mathbf{L}_{1}\langle\mathbf{e}\rangle_{1}+f_{2} \mathbf{L}_{2}\langle\mathbf{e}\rangle_{2}, \\
\langle\mathbf{p}\rangle & =f_{1}\langle\mathbf{p}\rangle_{1}+f_{2}\langle\mathbf{p}\rangle_{2} \\
& =f_{1} \boldsymbol{\rho}_{1}\langle\mathbf{v}\rangle_{1}+f_{2} \boldsymbol{\rho}_{2}\langle\mathbf{v}\rangle_{2}, \\
f_{1}+f_{2} & =1 .
\end{aligned}
$$

By eliminating $\langle\mathbf{e}\rangle_{2}$ and $\langle\mathbf{v}\rangle_{2}$ through (6) and (7), (8) and (9) can be rewritten as

$$
\begin{aligned}
& \langle\boldsymbol{\sigma}\rangle=\mathbf{L}_{2}\langle\mathbf{e}\rangle+f_{1}\left(\mathbf{L}_{1}-\mathbf{L}_{2}\right)\langle\mathbf{e}\rangle_{1}, \\
& \langle\mathbf{p}\rangle=\boldsymbol{\rho}_{2}\langle\mathbf{v}\rangle+f_{1}\left(\boldsymbol{\rho}_{1}-\boldsymbol{\rho}_{2}\right)\langle\mathbf{v}\rangle_{1},
\end{aligned}
$$

with

$$
\langle\bullet\rangle_{r}(\mathbf{x}, t)=\frac{1}{\left|\Omega_{r}\right|} \int_{U_{r}}(\bullet)\left(\mathbf{x}, t ; \mathbf{x}^{\prime}\right) \mathrm{d} \mathbf{x}^{\prime},
$$

where $U_{r}$ represents the region $\left\{\mathbf{x}^{\prime}: \mathbf{x}-\mathbf{x}^{\prime} \in \Omega_{r}\right\},\left|\Omega_{r}\right|$ represents the volume of $\Omega_{r}, r$ represents the $\mathrm{r}$-th phase material, $\mathbf{x}$ is the spatial coordinate, $t$ is time, and $\mathbf{x}^{\prime}$ is the center of the inclusion.

2.2. Single Inclusion Scattering Problem. The propagation of plane harmonic wave in composite materials is considered and has the form [24]:

$$
\mathbf{u}_{0}(\mathbf{x}, t)=\mathbf{m} \exp i(k \mathbf{n} \cdot \mathbf{x}-\omega t)
$$

where $\mathbf{u}_{0}$ denotes physical quantities such as stress $\boldsymbol{\sigma}$, strain $\mathbf{e}$, density $\boldsymbol{\rho}$, and velocity $\mathbf{v}, \mathbf{m}$ is the amplitude of these physical quantities, $i=\sqrt{-1}, k$ is the wave number, $\mathbf{n}$ is the unit directional vector of wave propagation, and $\omega$ is the circular frequency.

At the same time, polarization stress $\tau$ and polarization momentum $\pi$ are introduced with the form [30]:

$$
\begin{aligned}
& \boldsymbol{\tau}=\left(\mathbf{L}_{r}-\mathbf{L}_{0}\right) \mathbf{e}, \\
& \boldsymbol{\pi}=\left(\boldsymbol{\rho}_{r}-\boldsymbol{\rho}_{0}\right) \mathbf{v},
\end{aligned}
$$

where $r$ refers to $r$-th phase material, $r=1$ represents inclusion, $r=2$ represents matrix, $\mathbf{L}_{0}$ represents modulus of reference medium, and $\rho_{0}$ represents density of the reference medium. For the convenience of analysis, let the modulus and density of the reference medium be the same as the matrix material. Then, it can be known from (15) and (16) that the polarization stress and polarization momentum only exist in the inclusion but not in the matrix.

Then, stress $\boldsymbol{\sigma}$ and momentum $\mathbf{p}$ can be obtained from (15) and (16) with the following form:

$$
\begin{aligned}
& \boldsymbol{\sigma}=\mathbf{L}_{0} \mathbf{e}+\boldsymbol{\tau}, \\
& \mathbf{p}=\boldsymbol{\rho}_{0} \mathbf{v}+\boldsymbol{\pi} .
\end{aligned}
$$

Substituting (17) and (18) into (2) and taking (div $\tau-\dot{\pi})$ as the body force term, we have

$$
\mathbf{u}=\mathbf{u}_{0}+\mathbf{G} *(\operatorname{div} \boldsymbol{\tau}-\dot{\pi})=\mathbf{u}_{0}-\mathbf{S} * \boldsymbol{\tau}-\mathbf{M} * \boldsymbol{\pi},
$$

where $\mathbf{G}$ is the elastodynamic Green's function, $\mathbf{S}$ and $\mathbf{M}$ are the quantities related to Green's function, and * represents convolution operation. Specific expression of elastodynamic Green's function can be seen from equation (A.1) in Appendix A.

According to the study in [24] and in combination with (15) and (16), we have

$$
\begin{aligned}
& \left(\mathbf{L}_{1}-\mathbf{L}_{0}\right)^{-1} \boldsymbol{\tau}+\mathbf{S}_{\mathbf{x}} * \boldsymbol{\tau}+\mathbf{M}_{\mathbf{x}} * \boldsymbol{\pi}=\mathbf{e}_{0}, \\
& \left(\boldsymbol{\rho}_{1}-\boldsymbol{\rho}_{0}\right)^{-1} \boldsymbol{\pi}+\mathbf{S}_{t} * \boldsymbol{\tau}+\mathbf{M}_{t} * \boldsymbol{\pi}=\mathbf{v}_{0},
\end{aligned}
$$

where $\mathbf{S}_{\mathbf{x}}, \mathbf{M}_{\mathbf{x}}, \mathbf{S}_{t}$, and $\mathbf{M}_{t}$ are the quantities related to Green's function [24]. It can be verified that $\overline{\mathbf{M}}_{\mathbf{x}}=0$ and $\overline{\mathbf{S}}_{t}=0$ because the inclusion has a center of symmetry, where the bar above $\mathbf{M}_{\mathbf{x}}$ and $\mathbf{S}_{t}$ represents the volume average over the inclusion.

From (20) and (21), we have

$$
\begin{aligned}
& \boldsymbol{\tau}=\left[\left(\mathbf{L}_{1}-\mathbf{L}_{0}\right)^{-1}+\overline{\mathbf{S}}_{\mathbf{x}}\right]^{-1} \overline{\mathbf{e}}_{0}, \\
& \boldsymbol{\pi}=\left[\left(\boldsymbol{\rho}_{1}-\boldsymbol{\rho}_{0}\right)^{-1}+\overline{\mathbf{M}}_{t}\right]^{-1} \overline{\mathbf{v}}_{0},
\end{aligned}
$$

where the specific expressions of $\overline{\mathbf{S}}_{\mathbf{x}}$ and $\overline{\mathbf{M}}_{t}$ can be seen from equations (A.6) and (A.7) in Appendix A, respectively.

In combination with (15), (16), (22), and (23), we have

$$
\begin{aligned}
& \mathbf{e}=\left[\mathbf{I}+\overline{\mathbf{S}}_{\mathbf{x}}\left(\mathbf{L}_{1}-\mathbf{L}_{0}\right)\right]^{-1} \bar{e}_{0}, \\
& \mathbf{v}=\left[\mathbf{I}+\overline{\mathbf{M}}_{t}\left(\boldsymbol{\rho}_{1}-\boldsymbol{\rho}_{0}\right)\right]^{-1} \overline{\mathbf{v}}_{0} .
\end{aligned}
$$


Assuming that the center of the inclusion is at $\mathbf{x}^{\prime}$ and $\overline{\mathbf{e}}_{0}=\mathbf{e}_{0}\left(\mathbf{x}^{\prime}\right) h(k \mathbf{n})$, we obtain

$$
\begin{gathered}
\langle\mathbf{e}\rangle_{1}(\mathbf{x})=h(k \mathbf{n})\left[\mathbf{I}+\overline{\mathbf{S}}_{\mathbf{x}}\left(\mathbf{L}_{1}-\mathbf{L}_{0}\right)\right]^{-1} \frac{1}{|\Omega|} \int_{\Omega} \mathbf{e}_{0}\left(\mathbf{x}^{\prime}\right) \mathrm{d} \mathbf{x}^{\prime}, \\
\langle\mathbf{v}\rangle_{1}(\mathbf{x})=h(k \mathbf{n})\left[\mathbf{I}+\overline{\mathbf{M}}_{t}\left(\boldsymbol{\rho}_{1}-\boldsymbol{\rho}_{0}\right)\right]^{-1} \frac{1}{|\Omega|} \int_{\Omega} \mathbf{v}_{0}\left(\mathbf{x}^{\prime}\right) \mathrm{d} \mathbf{x}^{\prime},
\end{gathered}
$$

with

$$
h(k \mathbf{n})=\frac{1}{|\Omega|} \int_{\Omega} \exp (i k \mathbf{n} \cdot \mathbf{x}) \mathrm{d} \mathbf{x}
$$

Due to $\mathbf{e}_{0}\left(\mathbf{x}^{\prime}\right) \exp [i k \mathbf{n} \cdot \mathbf{x}]=\mathbf{e}_{0}(\mathbf{x}) \exp \left[i k \mathbf{n} \cdot \mathbf{x}^{\prime}\right], \mathbf{e}_{0}\left(\mathbf{x}^{\prime}\right)=$ $\mathbf{e}_{0}(\mathbf{x}) \exp \left[-i k \mathbf{n} \cdot\left(\mathbf{x}-\mathbf{x}^{\prime}\right)\right]$ is obtained, then,

$$
\begin{aligned}
\langle\mathbf{e}\rangle_{1}(\mathbf{x}) & =h(k \mathbf{n})\left[\mathbf{I}+\overline{\mathbf{S}}_{\mathbf{x}}\left(\mathbf{L}_{1}-\mathbf{L}_{0}\right)\right]^{-1} \frac{1}{|\Omega|} \int_{\Omega} \mathbf{e}_{0}\left(\mathbf{x}^{\prime}\right) \mathrm{d} \mathbf{x}^{\prime} \\
& =h(k \mathbf{n})\left[\mathbf{I}+\overline{\mathbf{S}}_{\mathbf{x}}\left(\mathbf{L}_{1}-\mathbf{L}_{0}\right)\right]^{-1} \frac{1}{|\Omega|} \int_{\Omega}\left\{\mathbf{e}_{0}(\mathbf{x}) \exp \left[-i k \mathbf{n} \cdot\left(\mathbf{x}-\mathbf{x}^{\prime}\right)\right]\right\} \mathrm{d} \mathbf{x}^{\prime} \\
& =h(k \mathbf{n})\left[\mathbf{I}+\overline{\mathbf{S}}_{\mathbf{x}}\left(\mathbf{L}_{1}-\mathbf{L}_{0}\right)\right]^{-1} \frac{1}{|\Omega|} \int_{\Omega}\left\{\mathbf{e}_{0}(\mathbf{x}) \exp \left[i k \mathbf{n} \cdot\left(\mathbf{x}^{\prime}-\mathbf{x}\right)\right]\right\} \mathrm{d}\left(\mathbf{x}^{\prime}-\mathbf{x}\right) \\
& =h(k \mathbf{n}) h(k \mathbf{n})\left[\mathbf{I}+\overline{\mathbf{S}}_{\mathbf{x}}\left(\mathbf{L}_{1}-\mathbf{L}_{0}\right)\right]^{-1} \mathbf{e}_{0}(\mathbf{x}) .
\end{aligned}
$$

Also, similarly,

$$
\langle\mathbf{v}\rangle_{1}(\mathbf{x})=h(k \mathbf{n}) h(k \mathbf{n})\left[\mathbf{I}+\overline{\mathbf{M}}_{t}\left(\boldsymbol{\rho}_{1}-\boldsymbol{\rho}_{0}\right)\right]^{-1} \mathbf{v}_{0}(\mathbf{x}) .
$$

Substituting (29) and (30) into (11) and (12), we have

$$
\begin{aligned}
\langle\boldsymbol{\sigma}\rangle & =\mathbf{L}_{2}\langle\mathbf{e}\rangle+f_{1}\left(\mathbf{L}_{1}-\mathbf{L}_{2}\right)\langle\mathbf{e}\rangle_{1} \\
& =\mathbf{L}_{2}\langle\mathbf{e}\rangle+f_{1}\left(\mathbf{L}_{1}-\mathbf{L}_{2}\right) h(k \mathbf{n}) h(k \mathbf{n})\left[\mathbf{I}+\overline{\mathbf{S}}_{\mathbf{x}}\left(\mathbf{L}_{1}-\mathbf{L}_{0}\right)\right]^{-1} \mathbf{e}_{0}, \\
\langle\mathbf{p}\rangle & =\boldsymbol{\rho}_{2}\langle\mathbf{v}\rangle+f_{1}\left(\boldsymbol{\rho}_{1}-\boldsymbol{\rho}_{2}\right)\langle\mathbf{v}\rangle_{1} \\
& =\boldsymbol{\rho}_{2}\langle\mathbf{v}\rangle+f_{1}\left(\boldsymbol{\rho}_{1}-\boldsymbol{\rho}_{2}\right) h(k \mathbf{n}) h(k \mathbf{n})\left[\mathbf{I}+\overline{\mathbf{M}}_{t}\left(\boldsymbol{\rho}_{1}-\boldsymbol{\rho}_{0}\right)\right]^{-1} \mathbf{v}_{0} .
\end{aligned}
$$

Let $\overline{\mathbf{L}}$ and $\overline{\boldsymbol{\rho}}$ represent the overall effective modulus and the overall effective density of the material, respectively. From equations (31) and (32), we have

$$
\begin{aligned}
\langle\boldsymbol{\sigma}\rangle & =\overline{\mathbf{L}}\langle\mathbf{e}\rangle \\
& =\mathbf{L}_{2}\langle\mathbf{e}\rangle+f_{1}\left(\mathbf{L}_{1}-\mathbf{L}_{2}\right) h(k \mathbf{n}) h(k \mathbf{n})\left[\mathbf{I}+\overline{\mathbf{S}}_{\mathbf{x}}\left(\mathbf{L}_{1}-\mathbf{L}_{0}\right)\right]^{-1} \mathbf{e}_{0}, \\
\langle\mathbf{p}\rangle & =\overline{\boldsymbol{\rho}}\langle\mathbf{v}\rangle \\
& =\boldsymbol{\rho}_{2}\langle\mathbf{v}\rangle+f_{1}\left(\boldsymbol{\rho}_{1}-\boldsymbol{\rho}_{2}\right) h(k \mathbf{n}) h(k \mathbf{n})\left[\mathbf{I}+\overline{\mathbf{M}}_{t}\left(\boldsymbol{\rho}_{1}-\boldsymbol{\rho}_{0}\right)\right]^{-1} \mathbf{v}_{0} .
\end{aligned}
$$

2.3. Differential Method. For the two-phased heterogeneous composite material, it is assumed that the volume fraction of inclusion is $f_{1}$ at some time. When the infinitesimal element of volume $\mathrm{d} V$ is removed and the same volume of inclusion is added, the volume fraction of inclusion is $f_{1}+\mathrm{d} f_{1}$. Then, the volume of inclusion in the new composite is

$$
f_{1} V+\mathrm{d} V-f_{1} \mathrm{~d} V=\left(f_{1}+\mathrm{d} f_{1}\right) V .
$$

Equation (35) can be simplified as [31]

$$
\frac{\mathrm{d} V}{V}=\frac{\mathrm{d} f_{1}}{1-f_{1}} .
$$

When the volume fraction of inclusion is $f_{1}$ in composite materials at some time, let the modulus and density of the composite be $\overline{\mathbf{L}}\left(f_{1}\right)$ and $\overline{\boldsymbol{\rho}}\left(f_{1}\right)$, respectively. Then, let the composite at this time be as matrix, namely, $\mathbf{L}_{2}=\overline{\mathbf{L}}\left(f_{1}\right)$ and $\boldsymbol{\rho}_{2}=\overline{\boldsymbol{\rho}}\left(f_{1}\right)$. After adding the volume $\mathrm{d} V$ of inclusion, let the overall effective modulus be $\overline{\mathbf{L}}\left(f_{1}+\mathrm{d} f_{1}\right)$ and density be $\overline{\boldsymbol{\rho}}\left(f_{1}+\mathrm{d} f_{1}\right)$. By replacing $\mathbf{L}_{2}, \boldsymbol{\rho}_{2}, \overline{\mathbf{L}}$, and $\overline{\boldsymbol{\rho}}$ in equations (33) and (34) by $\overline{\mathbf{L}}\left(f_{1}\right), \overline{\boldsymbol{\rho}}\left(f_{1}\right), \overline{\mathbf{L}}\left(f_{1}+\mathrm{d} f_{1}\right)$, and $\overline{\boldsymbol{\rho}}\left(f_{1}+\mathrm{d} f_{1}\right)$, respectively, equations (33) and (34) can be rewritten as

$$
\begin{aligned}
& {\left[\overline{\mathbf{L}}\left(f_{1}+\mathrm{d} f_{1}\right)-\overline{\mathbf{L}}\left(f_{1}\right)\right]\langle\mathbf{e}\rangle=\frac{\mathrm{d} V}{V}\left(\mathbf{L}_{1}-\overline{\mathbf{L}}\left(f_{1}\right)\right) h(k \mathbf{n}) h(k \mathbf{n})\left[\mathbf{I}+\overline{\mathbf{S}}_{\mathbf{x}}\left(f_{1}\right)\left(\mathbf{L}_{1}-\overline{\mathbf{L}}\left(f_{1}\right)\right)\right]^{-1} \mathbf{e}_{0},} \\
& {\left[\overline{\boldsymbol{\rho}}\left(f_{1}+\mathrm{d} f_{1}\right)-\overline{\boldsymbol{\rho}}\left(f_{1}\right)\right]\langle\mathbf{v}\rangle=\frac{\mathrm{d} V}{V} h(k \mathbf{n}) h(k \mathbf{n})\left(\boldsymbol{\rho}_{1}-\overline{\boldsymbol{\rho}}\left(f_{1}\right)\right)\left[\mathbf{I}+\overline{\mathbf{M}}_{t}\left(f_{1}\right)\left(\boldsymbol{\rho}_{1}-\overline{\boldsymbol{\rho}}\left(f_{1}\right)\right)\right]^{-1} \mathbf{v}_{0},}
\end{aligned}
$$


When $\mathrm{d} V \longrightarrow 0[32-34]$, we have

$$
\begin{aligned}
& \langle\mathbf{e}\rangle=\mathbf{e}_{0}\left[1+\mathrm{O}\left(\frac{\mathrm{d} V}{V}\right)\right], \\
& \langle\mathbf{v}\rangle=\mathbf{v}_{0}\left[1+\mathrm{O}\left(\frac{\mathrm{d} V}{V}\right)\right] .
\end{aligned}
$$

In combination with (36), (39), and (40), equations (37) and (38) can be rearranged as

$$
\begin{aligned}
& \frac{\mathrm{d} \overline{\mathbf{L}}\left(f_{1}\right)}{\mathrm{d} f_{1}}=\frac{1}{1-f_{1}} h(k \mathbf{n}) h(k \mathbf{n})\left(\mathbf{L}_{1}-\overline{\mathbf{L}}\left(f_{1}\right)\right)\left[\mathbf{I}+\overline{\mathbf{S}}_{\mathbf{x}}\left(f_{1}\right)\left(\mathbf{L}_{1}-\overline{\mathbf{L}}\left(f_{1}\right)\right)\right]^{-1}, \\
& \frac{\mathrm{d} \overline{\boldsymbol{\rho}}\left(f_{1}\right)}{\mathrm{d} f_{1}}=\frac{1}{1-f_{1}} h(k \mathbf{n}) h(k \mathbf{n})\left(\boldsymbol{\rho}_{1}-\overline{\boldsymbol{\rho}}\left(f_{1}\right)\right)\left[\mathbf{I}+\overline{\mathbf{M}}_{t}\left(f_{1}\right)\left(\boldsymbol{\rho}_{1}-\overline{\boldsymbol{\rho}}\left(f_{1}\right)\right)\right]^{-1} .
\end{aligned}
$$

The initial conditions of the corresponding expressions of (37) and (38) are, respectively,

$$
\begin{aligned}
& \left.\overline{\mathbf{L}}\left(f_{1}\right)\right|_{\mathbf{f}_{1}=0}=\mathbf{L}_{2}, \\
& \left.\overline{\boldsymbol{\rho}}\left(f_{1}\right)\right|_{\mathbf{f}_{1}=0}=\boldsymbol{\rho}_{2} .
\end{aligned}
$$

\section{Results and Discussion}

For the convenience of obtaining numerical results for quantitative analysis, equations (41) (44) are numerically solved by the fourth-order Runge-Kutta method [35]. Specific formations of equations (41) (44) can be seen from equations (B.24) to (B.25) in Appendix B. The iteration step length $h$ is 0.001 , and the calculation formula of phase velocity can be seen from equation (B.13). Material properties are same with the matrix when calculating the phase velocity of the matrix material, and material properties are taken as dynamic effective properties when calculating phase velocity of the composite. The matrix is epoxy, and inclusion is lead. Material's properties are shown in Table 1 [5]:

3.1. Verification of the Differential Method. For $\delta=1$, quasi$\mathrm{P}$ wave propagation is calculated by the differential method and self-consistent method for inclusion volume fractions of $5.4 \%$ and $15.9 \%$, respectively. The ratio of the phase velocity in the composite and matrix is compared with the experimental results and shown in Figures 2 and 3 below. For $\delta=0.2$, quasi-P wave propagation is obtained by the differential method and self-consistent method, respectively, for inclusion volume fraction of $1 \%$. The ratio of the phase velocity in the composite and matrix is shown in Figure 4.

As can be seen from Figure 2, when the inclusion volume fraction is $5.4 \%$, the ratio of the phase velocity calculated by the differential method is in good agreement with the results obtained by experiments as well as the self-consistent method. Figure 3 shows that when the inclusion volume fraction is $15.9 \%$ and the dimensionless wave number $\omega \mathrm{a} / \mathrm{c}_{1}$ is at $0.3-0.5$, the ratio of the phase velocity obtained by the differential method is larger than the results obtained by the self-consistent method. This is mainly due to the influence of resonance area included in the range of $0.3-0.5$, and in other areas, the results obtained by the differential method are in good agreement with the self-consistent method and experiments. As can be seen from Figure 4, when $\delta=0.2$ and the inclusion volume fraction is $1 \%$, the results obtained by the differential method are in good agreement with the selfconsistent method. Figures 2-4 also verify the correctness of results obtained by the differential method.

3.2. Comparisons of Different Forms of Inclusion with the Differential Method and Self-Consistent Method. When $\delta=0.5$ and $\delta=5$, the differential method and self-consistent method are used to calculate the phase velocity ratio of quasi-P wave propagation in the composite material for inclusion volume fraction of $5.4 \%$ and $15.9 \%$, respectively, and the comparisons of the phase velocity ratio are shown in Figures 5 and 6.

Figures 5 and 6 show that when the inclusion volume fraction is $5.4 \%$, results obtained by the differential method are in good agreement with the self-consistent method. Phase velocity ratio near the resonance zone obtained by the differential method is larger than the self-consistent method when $\delta=0.5$, and phase velocity ratio near the resonance zone obtained by the self-consistent method is larger than the differential method when $\delta=5$.

When $\delta=0.5,1$, and 5 , the differential method is used to calculate the phase velocity ratio of quasi-P wave propagation in the composite material with inclusion volume fraction of $5.4 \%$ and $15.9 \%$, respectively. Results are shown in Figures 7 and 8.

It can be seen from Figure 8 that when the inclusion volume fraction is $15.9 \%$, the peak value of the phase velocity ratio becomes small as the value of $\delta$ increases.

Figures 2-8 show that when the dimensionless wave number $\omega \mathrm{a} / \mathrm{c}_{1}$ is large, the value of the ratio of the phase velocity in the composite and matrix tends to 1 . This is because when $\omega \mathrm{a} / \mathrm{c}_{1}$ takes a larger value, according to equation (A.14) in Appendix A, we have $h(k \mathbf{n}) h(k \mathbf{n}) \longrightarrow 0$, and this leads to the right side of equations (41) and (42) 
TABLE 1: Elastic modulus and density of the matrix and inclusion. The units of modulus and density are $\mathrm{GPa}$ and $10^{3} \mathrm{Kg} / \mathrm{m}^{3}$, respectively.

\begin{tabular}{lccccccc}
\hline Materials & $\rho$ & $L_{1111}$ & $L_{1122}$ & $L_{1133}$ & $L_{3333}$ & $L_{2323}$ & $L_{1212}$ \\
\hline Epoxy & 1.202 & 8.377 & 4.915 & 4.915 & 8.377 & 1.731 & 1.731 \\
Lead & 11.3 & 55.4598 & 38.7598 & 38.7598 & 55.4598 & 8.35 & 8.35 \\
\hline
\end{tabular}

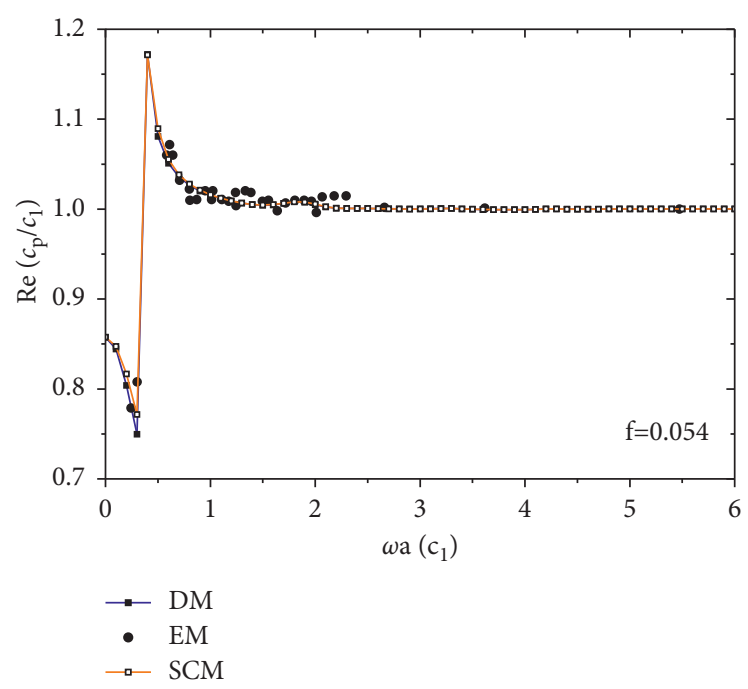

Figure 2: Comparison of ratios of the phase velocity in the composite material and matrix obtained by the differential method (DM) and self-consistent method (SCM), and experimental results (ER) in the case of quasi-P wave propagates in the composite material for inclusion volume fraction of $5.4 \%$ and $\delta=1$.

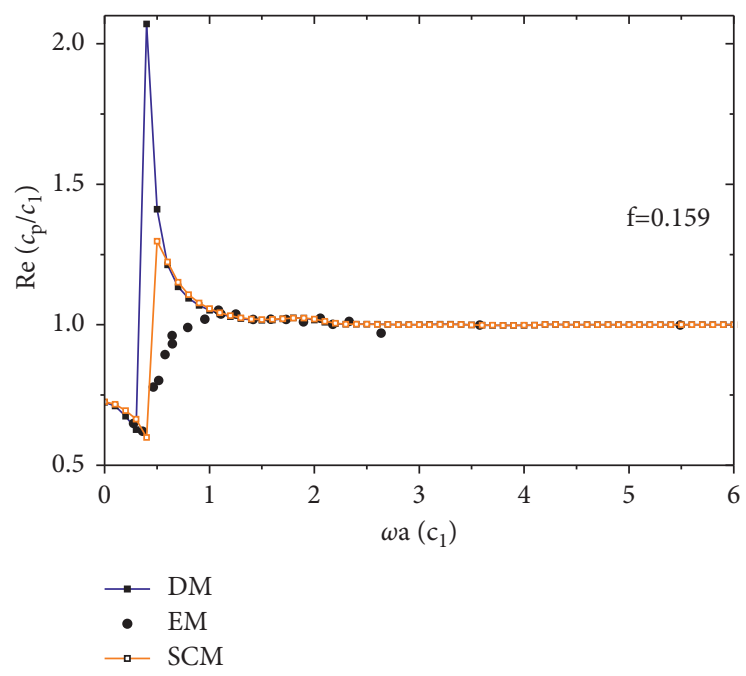

Figure 3: Comparison of ratios of the phase velocity in the composite material and matrix obtained by the differential method (DM) and self-consistent method (SCM), and experimental results (ER) in the case of quasi-P wave propagates in the composite material for inclusion volume fraction of $15.9 \%$ and $\delta=1$.

tends to zero, namely, the dynamic effective properties of the composite tend to be same as matrix.

3.3. Dynamic Effective Properties of the Composites with the Differential Method and Self-Consistent Method. The differential method and self-consistent method are used to calculate the dynamic effective modulus and density of quasi-P wave propagation in the composite material for inclusion volume fractions of $5.4 \%$ and $15.9 \%$ when $\delta=0.5$, 1 , and 5, respectively. The dynamic effective density $\boldsymbol{\rho}_{11}$ and $\boldsymbol{\rho}_{33}$ and modulus $\mathbf{L}_{1111}, \mathbf{L}_{1122}, \mathbf{L}_{1133}$, and $\mathbf{L}_{3333}$ are shown in Figures 9 and 10. For the convenience of analysis and discussion, the real part of the obtained results is taken as the dynamic effective density and modulus.

According to Figures 9 and 10, the dynamic effective densities obtained by the differential method are larger than those obtained by the self-consistent method near the 


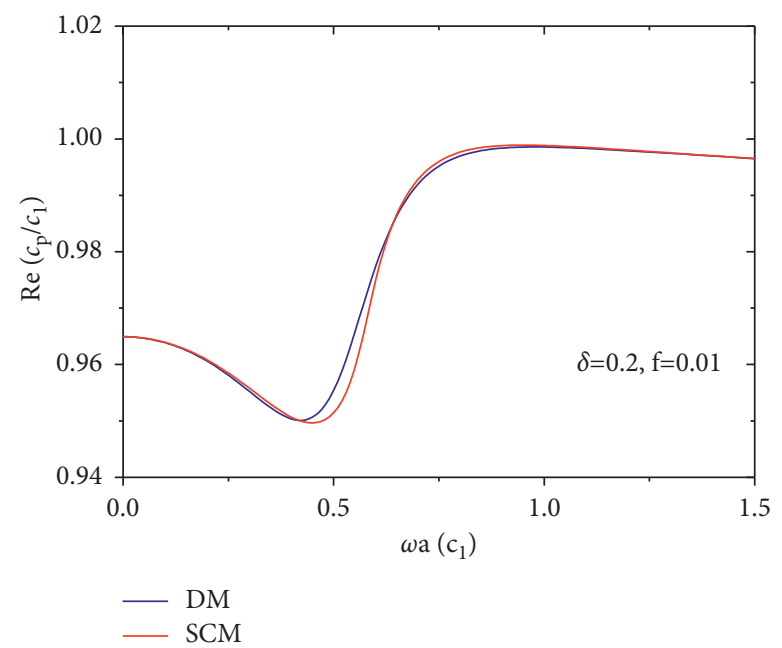

FIGURE 4: Comparison of ratios of the phase velocity in the composite material and matrix obtained by the differential method (DM) and self-consistent method (SCM) when quasi-P wave propagates in the composite material for inclusion volume fraction of $1 \%$ and $\delta=0.2$.

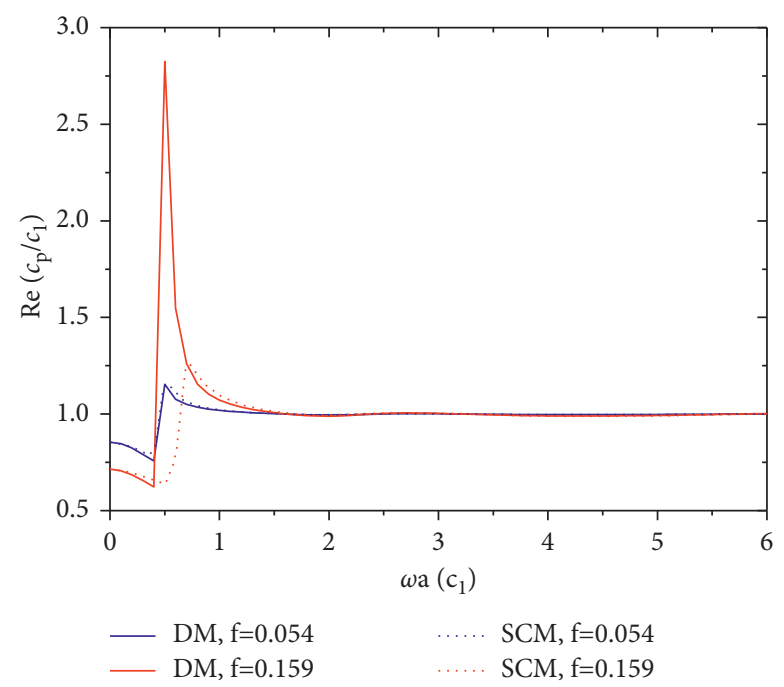

Figure 5: Comparison of ratios of the phase velocity in the composite material and matrix obtained by the differential method (DM) and self-consistent method (SCM) when quasi-P wave propagates in the composite material for inclusion volume fraction of $5.4 \%$ and $15.9 \%$ and $\delta=0.5$.

resonance zone when $\delta=0.5$ and 1 , and this means the results obtained by the differential method are more accurate.

According to Figures 9 and 10, the dynamic effective modulus $\mathbf{L}_{1111}$ and $\mathbf{L}_{1122}$ decreases with the increase of $\delta$. From Figures 9 and 10, the dynamic effective modulus $\mathbf{L}_{1133}$ and $\mathbf{L}_{3333}$ increases with $\delta$ increases when $\omega \mathrm{a} / \mathrm{c}_{1}$ is small. This is because the cross-section geometry of inclusion increases as $\delta$ increases in parallel to the direction of $\mathbf{x}_{3}$. Also, when $\omega \mathrm{a} / \mathrm{c}_{1}$ is small, especially when $\omega \mathrm{a} / \mathrm{c}_{1} \longrightarrow 0$, which can be thought as quasi-static condition, the results are in line with the actual case at this time.

As can be seen from Figures 9 and 10, when $\delta=1$, the matrix and inclusion are isotropic materials, and the dynamic effective density and modulus are also isotropic. When $\delta \neq 1$, although the matrix and inclusion are isotropic, the dynamic effective density and modulus are not isotropic. 


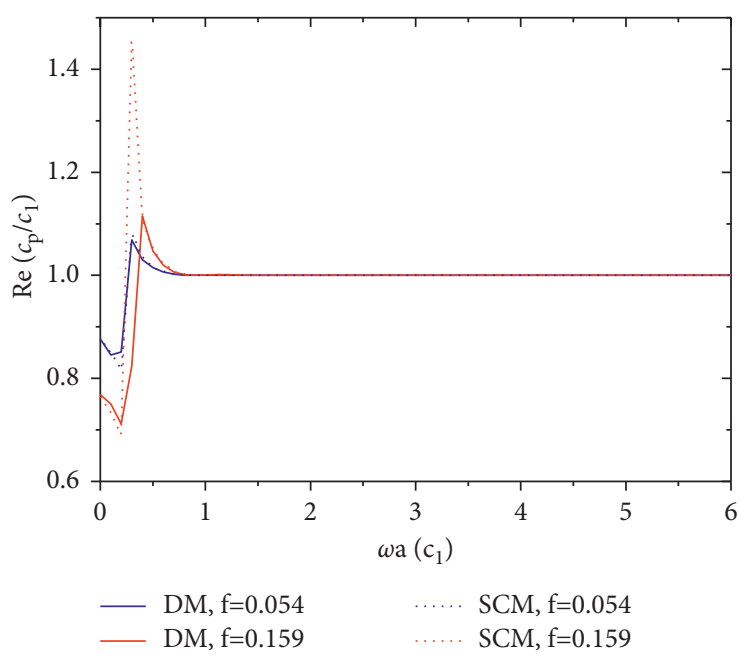

Figure 6: Comparison of ratios of the phase velocity in the composite material and matrix obtained by the differential method (DM) and self-consistent method (SCM) when quasi-P wave propagates in the composite material for inclusion volume fraction of $5.4 \%$ and $15.9 \%$ and $\delta=5$.

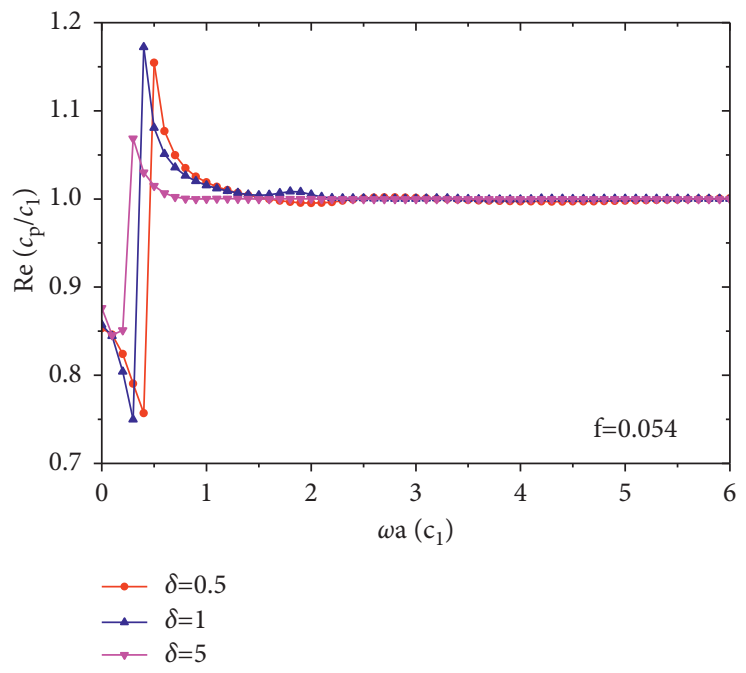

Figure 7: Comparison of ratios of the phase velocity in the composite material and matrix obtained by the differential method when quasi-P wave propagates in the composite material for inclusion volume fraction of $5.4 \%$ and $\delta=0.5,1$, and 5 , respectively.

This is because when $\delta=1$, the inclusions are spherical particles with highly symmetric of geometry, but when $\delta \neq 1$, the shape of the inclusion is rotating ellipsoid $(\delta>1)$ and flat $(\delta<1)$, respectively, and the geometry of the inclusions is not symmetrical. Therefore, even if the matrix and inclusion are isotropic, the dynamic effective density and modulus obtained are not isotropic when $\delta \neq 1$. Furthermore, the dynamic effective density and modulus tend to be same for different $\delta$ when the dimensionless wave number $\omega \mathrm{a} / \mathrm{c}_{1}$ is large enough. 


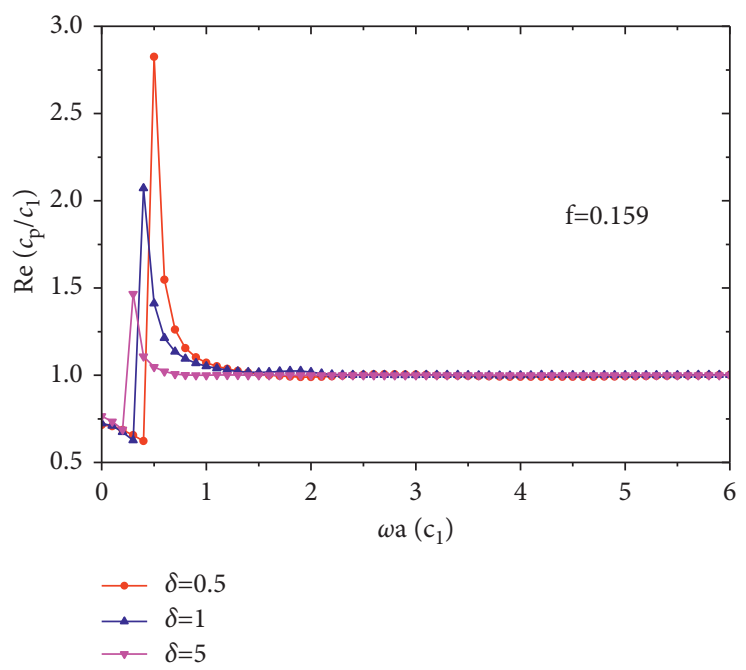

FIGURE 8: Comparison of ratios of the phase velocity in the composite material and matrix obtained by the differential method when quasi-P wave propagates in the composite material for inclusion volume fraction of $15.9 \%$ and $\delta=0.5,1$, and 5 , respectively.

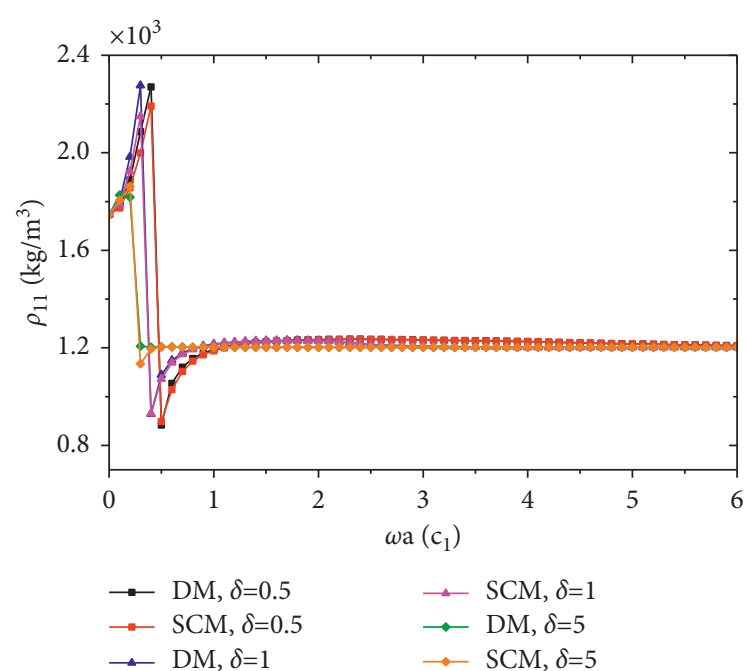

(a)

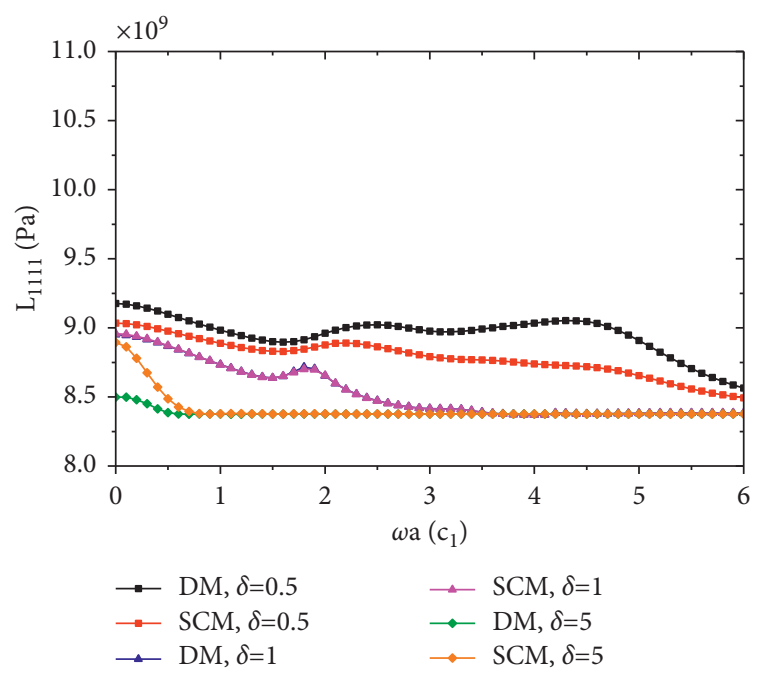

(c)

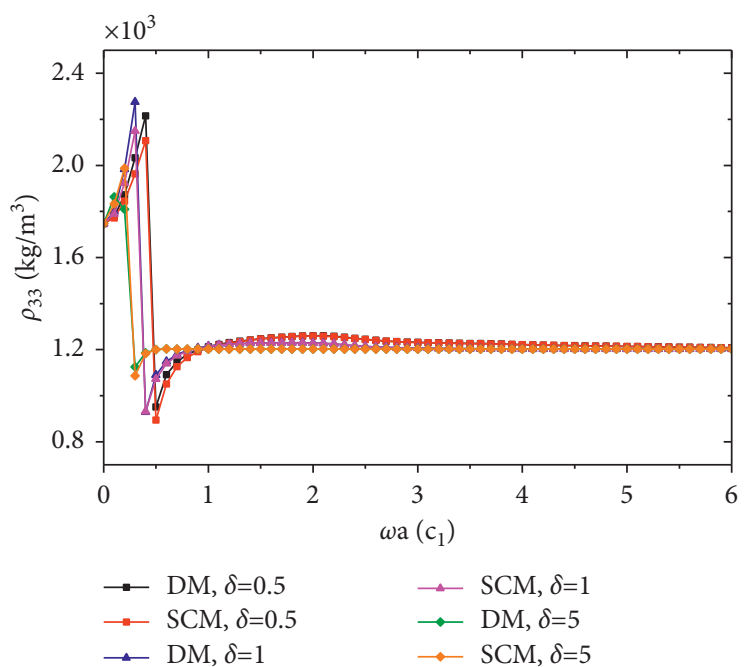

(b)

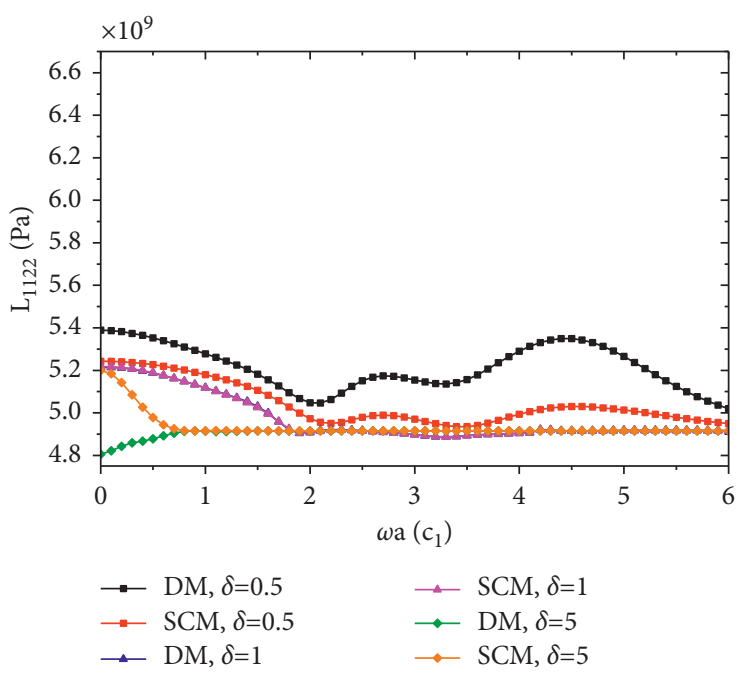

(d)

Figure 9: Continued. 


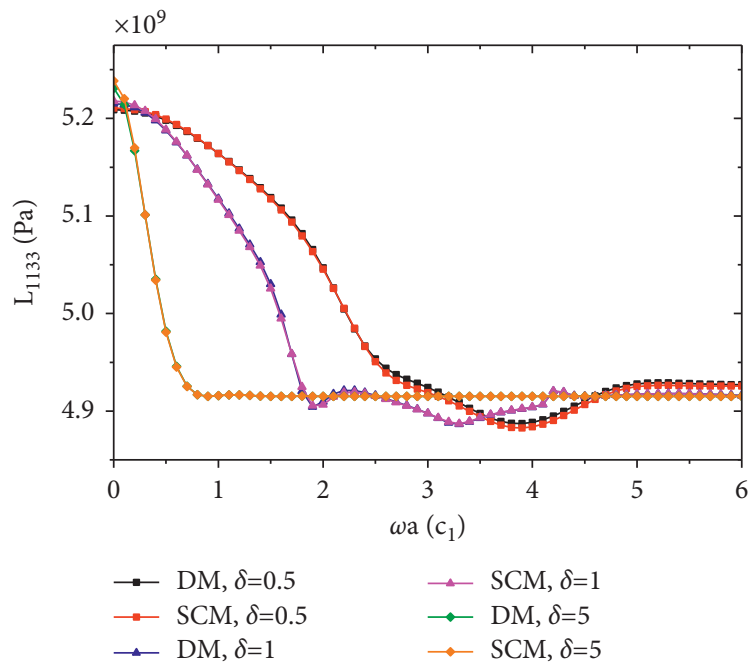

(e)

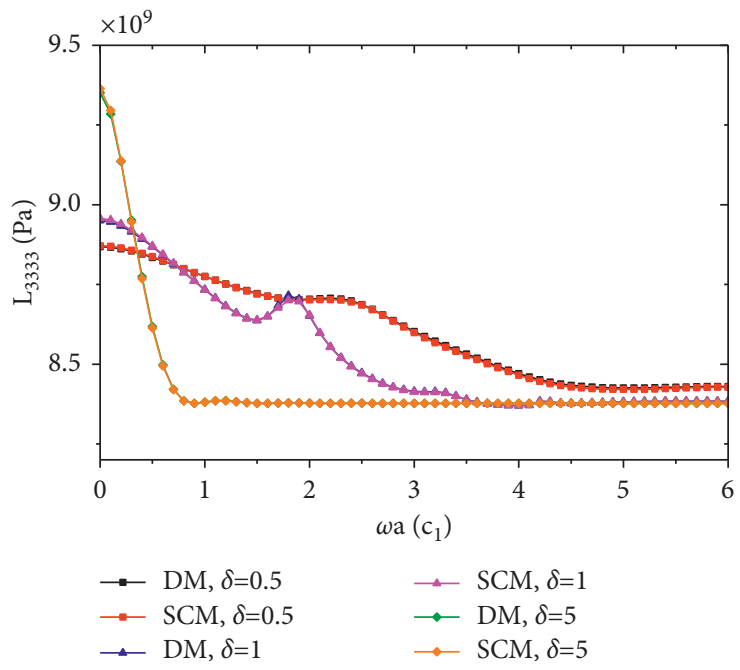

(f)

FigURE 9: When quasi-P wave propagates in the composite material for inclusion volume fraction of 5.4\% and $\delta=0.5,1$, and 5 , the dynamic effective density $\rho_{11}$ and $\rho_{33}$ and dynamic effective modulus $L_{1111}, L_{1122}, L_{1133}$, and $L_{3333}$ are obtained by the differential method (DM) and self-consistent method (SCM), respectively.

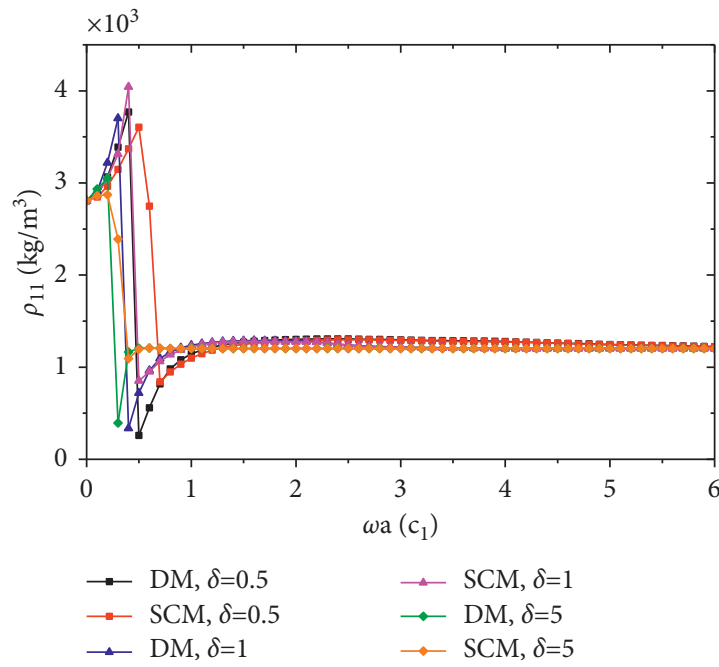

(a)

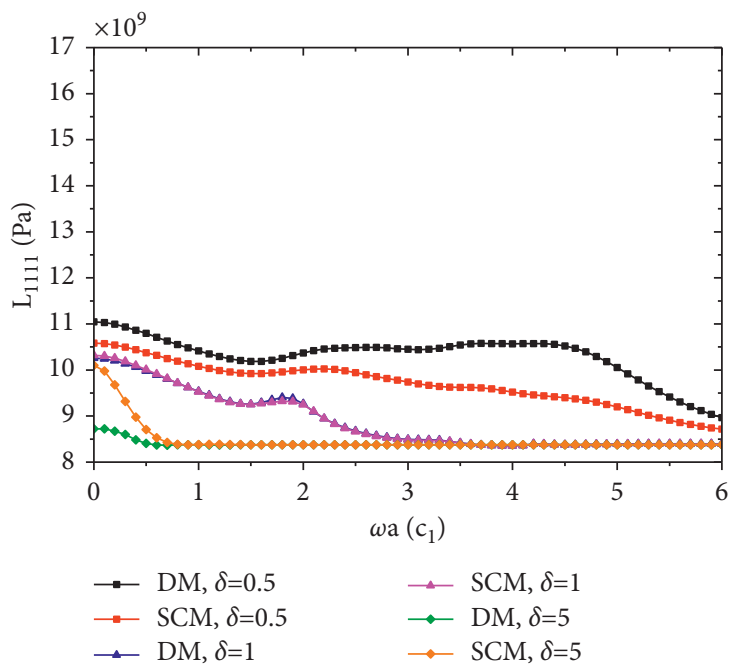

(c)

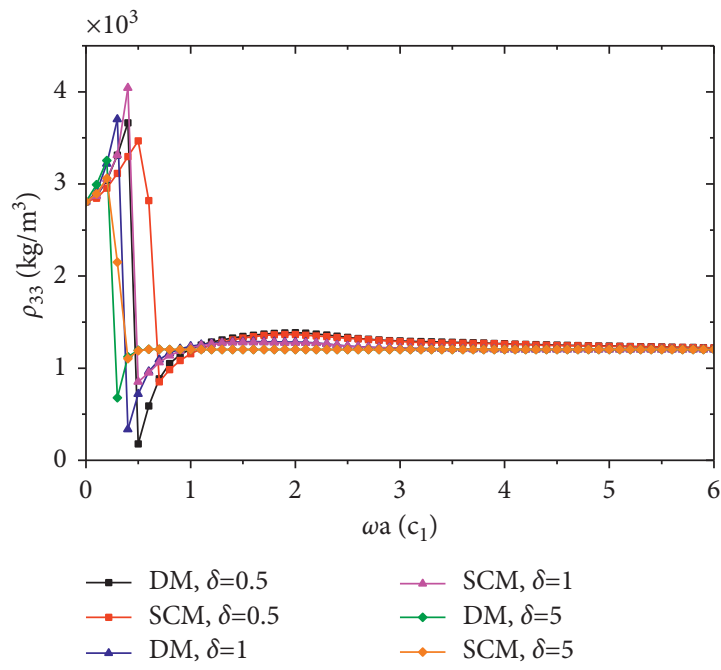

(b)

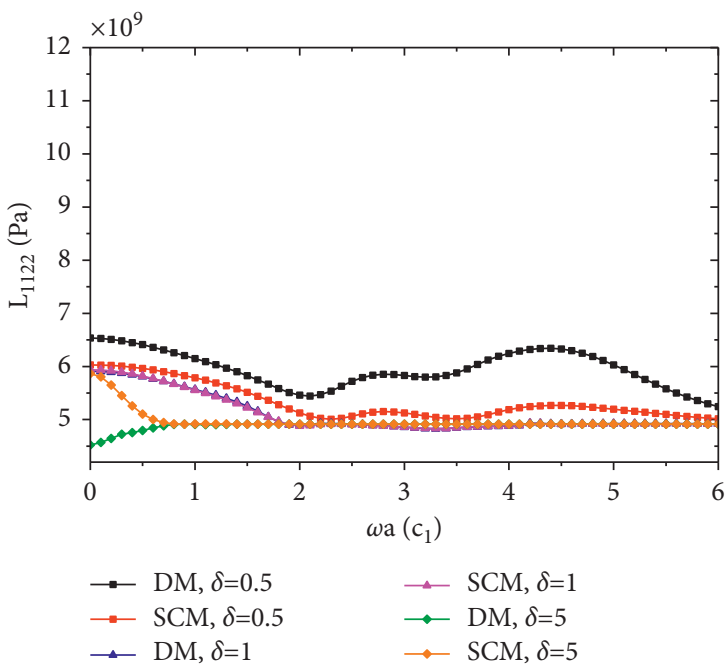

(d)

Figure 10: Continued. 


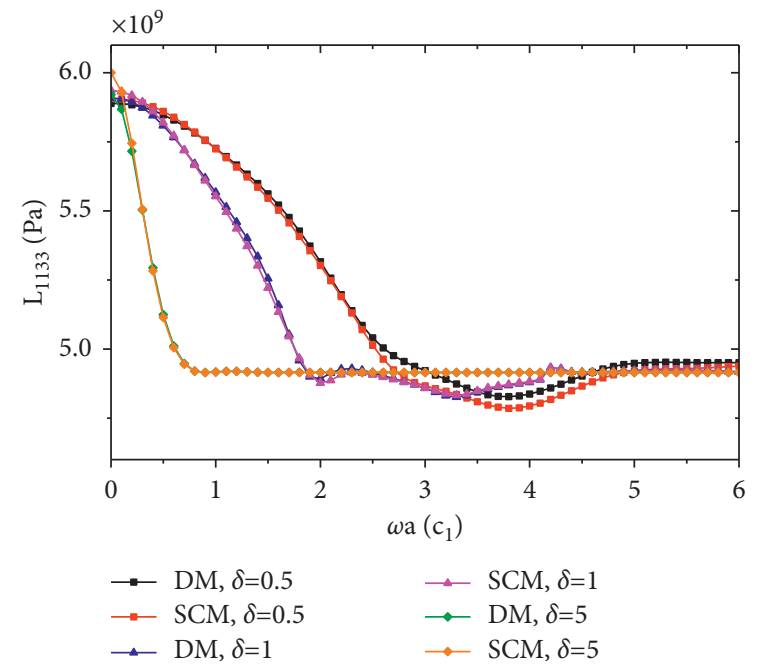

(e)

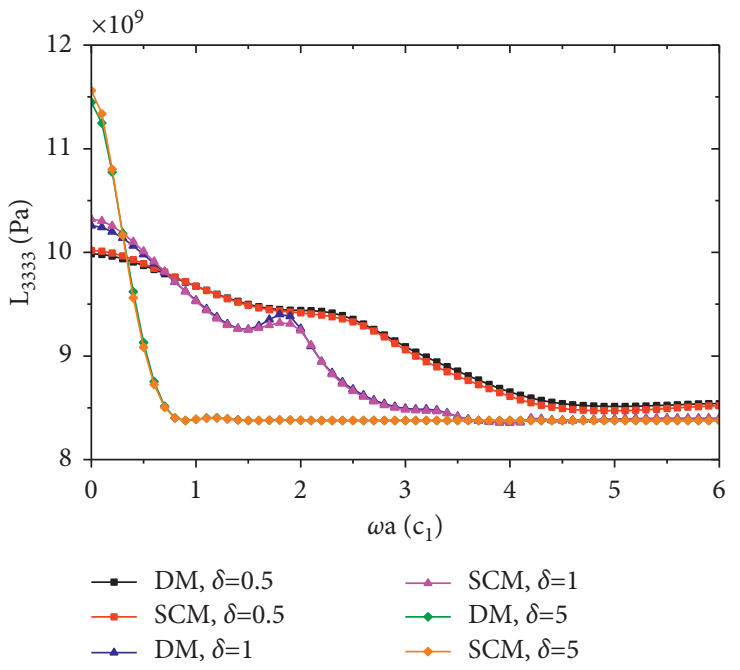

(f)

FIGURE 10: When quasi-P wave propagates in the composite material for inclusion volume fraction of $15.9 \%$ and $\delta=0.5,1$, and 5 , the dynamic effective density $\rho_{11}$ and $\rho_{33}$ and dynamic effective modulus $L_{1111}, L_{1122}, L_{1133}$, and $L_{3333}$ are obtained by the differential method (DM) and self-consistent method (SCM), respectively.

\section{Conclusions}

In this paper, the dynamic effective properties of a twophased composite with quasi-P wave propagation are solved by the differential method, and the results are compared with the experimental and theoretical results. From the results, we have as follows.

(1) The results obtained by the differential method are compared with the self-consistent method and experimental results, and the correctness of the results obtained by the differential method is verified.

(2) When $\delta=0.5$ and $\delta=1$, the peak value of the phase velocity obtained by the differential method is slightly higher than the value obtained by the selfconsistent method near the resonance region, which is more accurate.

(3) When the dimensionless wave number $\omega \mathrm{a} / \mathrm{c}_{1}$ is large, the phase velocity ratio of the quasi-P wave propagation in the composites tends to 1 .

(4) When $\delta \neq 1$, even if the inclusion material and the matrix material are isotropic, the dynamic effective density and modulus obtained are not isotropic, which is mainly due to the geometric shape of the inclusion.

(5) The dynamic effective modulus $\mathbf{L}_{1111}$ and $\mathbf{L}_{1122}$ decreases with the increase of $\delta$, and $\mathbf{L}_{1133}$ and $\mathbf{L}_{3333}$ increases with the increase of $\delta$.

\section{Appendix}

$\mathbf{A}$

The time-reduced elastodynamic Green's function of the reference medium is [24]

$$
\begin{aligned}
G_{i j}(\mathbf{x})= & \frac{1}{8 \pi^{2}} \sum_{N=1}^{3} \int_{|\xi|=1} \mathrm{~d} s \frac{U_{i}^{N}(\boldsymbol{\xi}) U_{j}^{N}(\boldsymbol{\xi})}{c_{N}^{2}} \\
& \left\{\delta(\xi \cdot \mathbf{x})+\frac{i \omega}{2 c_{N}} \exp \left(\frac{i \omega|\boldsymbol{\xi} \cdot \mathbf{x}|}{c_{N}}\right)\right\},
\end{aligned}
$$

where $U_{i}^{N}$ and $c_{N}$ are the polarization and wave speed of a plane wave propagation in the direction of the unit vector $\xi$, with $\xi_{i}=(\sin \theta \cos \phi$, sin $\theta \sin \phi, \cos \theta)$ and $\xi_{3}=\cos \theta$ $=u$, and $\delta(\xi \cdot \mathbf{x})$ is the three-dimensional delta-function.

The functions $\mathbf{S}_{\mathbf{x}}$ and $\mathbf{M}_{t}$ are related to equation (A.1) as

$$
\begin{aligned}
& \left(\mathbf{S}_{\mathbf{x}}\right)_{i j k l}=-\left.G_{i k, l j}\right|_{(i j),(k l)}, \\
& \left(\mathbf{M}_{t}\right)_{i j}=-\omega^{2} G_{i j} .
\end{aligned}
$$

Then, $\overline{\mathbf{S}}_{\mathbf{x}}$ and $\overline{\mathbf{M}}_{t}$ are defined by

$$
\begin{gathered}
\overline{\mathbf{S}}_{\mathbf{x}}=\frac{1}{\Omega} \iint_{\Omega \times \Omega} \mathbf{S}_{\mathbf{x}}(\mathbf{x}-\mathbf{y}) \mathrm{d} \mathbf{x} \mathrm{d} \mathbf{y}, \\
\overline{\mathbf{M}}_{t}=-\frac{\omega^{2}}{\Omega} \iint_{\Omega \times \Omega} \mathbf{G}(\mathbf{x}-\mathbf{y}) \mathrm{d} \mathbf{x} \mathrm{d} \mathbf{y} .
\end{gathered}
$$

Specific formations of $\overline{\mathbf{S}}_{\mathbf{x}}$ and $\overline{\mathbf{M}}_{t}$ are

$$
\left(\overline{\mathbf{S}}_{\mathbf{x}}\right)_{i j k l}=\left.\frac{1}{4 \pi} \sum_{N=1}^{3} \int_{|\xi|=1} \mathrm{~d} s\left\{\xi_{j} U_{i}^{N}(\xi) U_{k}^{N}(\xi) \xi_{l}\right\}\right|_{(i j)(k l)} H_{N}(\zeta(\xi)),
$$

$\left(\overline{\mathbf{M}}_{t}\right)_{i j}=\frac{1}{4 \pi} \sum_{N=1}^{3} \int_{|\xi|=1} d s U_{i}^{N}(\xi) U_{j}^{N}(\xi) F_{N}(\zeta(\xi))$,

where 


$$
\begin{aligned}
F_{N}(\zeta) & =\delta \cdot\left[1-\varepsilon\left(k_{N} a \zeta\right)\right] \cdot \zeta^{-3}, \\
H_{N}(\zeta) & =\delta \cdot \varepsilon\left(k_{N} a \zeta\right) c_{N}^{-2} \cdot \zeta^{-3}, \\
\zeta(\xi) & =\sqrt{\xi_{1}^{2}+\xi_{2}^{2}+\delta^{2} \xi_{3}^{2}}
\end{aligned}
$$

with

$$
\mathcal{E}(z)=\frac{3(1-i z)}{z^{3}} e^{i z}(\sin z-z \cos z) .
$$

The function $h(k \mathbf{n})$ is

$$
h(k \mathbf{n})=\hat{h}\left(k a \zeta\left(n_{3}\right)\right)
$$

where

$$
\widehat{h}(z)=\frac{3(\sin z-z \cos z)}{z^{3}},
$$

when $z \longrightarrow \infty$, according to L' Hopital's rule, we have

$$
\begin{aligned}
\lim _{z \longrightarrow \infty} \hat{h}(z) & =\lim _{z \longrightarrow \infty} \frac{3(\cos z-\cos z+z \sin z)}{3 z^{2}} \\
& =\lim _{z \longrightarrow \infty} \frac{\sin z}{z}
\end{aligned}
$$

$$
=0 \text {. }
$$

\section{B}

According to literature [24] and $\boldsymbol{\sigma}=\mathbf{L e}$, let $k=\mathbf{L}_{1111}+\mathbf{L}_{1122} / 2, \quad l=\mathbf{L}_{1133}, \quad q=\mathbf{L}_{3311}, \quad n=\mathbf{L}_{3333}$, $m=\mathbf{L}_{1111}+\mathbf{L}_{1122} / 2, p=\mathbf{L}_{2323}$, and the inclusion modulus tensor and dynamic effective modulus were denoted as $\mathbf{L}_{1}$ and $\overline{\mathbf{L}}$, respectively, so that [36]

$$
\begin{aligned}
\mathbf{L}_{1} & =\left(2 k_{1}, l_{1}, q_{1}, n_{1}, 2 m_{1}, 2 p_{1}\right), \\
\overline{\mathbf{L}} & =(2 \bar{k}, \bar{l}, \bar{q}, \bar{n}, 2 \bar{m}, 2 \bar{p}), \\
\mathbf{L}_{1}-\overline{\mathbf{L}} & =\left(2\left(k_{1}-\bar{k}\right), l_{1}-\bar{l}, q_{1}-\bar{q}, n_{1}-\bar{n}, 2\left(m_{1}-\bar{m}\right), 2\left(p_{1}-\bar{p}\right)\right) . \\
\overline{\mathbf{S}}_{\mathbf{x}} & =\left(2 k_{s}, l_{s}, q_{s}, n_{s}, 2 m_{s}, 2 p_{s}\right) .
\end{aligned}
$$

Let where the specific forms of $\overline{\mathbf{S}}_{\mathbf{x}}$ are

$$
\begin{aligned}
2 k_{s} & =\frac{1}{2 \boldsymbol{\rho}_{1}} \int_{0}^{1} \mathrm{~d} u\left(1-u^{2}\right)\left[m_{1}^{2} H_{1}(\zeta(u))+m_{3}^{2} H_{3}(\zeta(u))\right], \\
l_{s} & =q_{s} \\
& =\frac{1}{2 \sqrt{\boldsymbol{\rho}_{1} \boldsymbol{\rho}_{3}}} \int_{0}^{1} \mathrm{~d} u \sqrt{1-u^{2}} u m_{1} m_{3}\left[H_{1}(\zeta(u))-H_{3}(\zeta(u))\right], \\
n_{s} & =\frac{1}{\boldsymbol{\rho}_{3}} \int_{0}^{1} \mathrm{~d} u u^{2}\left[m_{3}^{2} H_{1}(\zeta(u))+m_{1}^{2} H_{3}(\zeta(u))\right], \\
2 m_{s} & =\frac{1}{4 \boldsymbol{\rho}_{1}} \int_{0}^{1} \mathrm{~d} u\left(1-u^{2}\right)\left[m_{1}^{2} H_{1}(\zeta(u))+H_{2}(\zeta(u))+m_{3}^{2} H_{3}(\zeta(u))\right], \\
2 p_{s}= & \frac{1}{4} \int_{0}^{1} \mathrm{~d} u\left\{\begin{array}{cc}
{\left[u^{2} \frac{m_{1}^{2}}{\boldsymbol{\rho}_{1}}+\left(1-u^{2}\right) \frac{m_{3}^{2}}{\boldsymbol{\rho}_{3}}+2 u \sqrt{1-u^{2}}\right.} & \left.\frac{m_{1} m_{3}}{\sqrt{\boldsymbol{\rho}_{1} \boldsymbol{\rho}_{3}}}\right] H_{1}(\zeta(u)) \\
+\left[u^{2} \frac{m_{3}^{2}}{\boldsymbol{\rho}_{1}}+\left(1-u^{2}\right) \frac{m_{1}^{2}}{\boldsymbol{\rho}_{3}}-2 u \sqrt{1-u^{2}}\right. & \left.\frac{m_{1} m_{3}}{\sqrt{\boldsymbol{\rho}_{1} \boldsymbol{\rho}_{3}}}\right] H_{3}(\zeta(u))
\end{array}\right\}
\end{aligned}
$$




$$
\begin{aligned}
& m_{1}=\sqrt{\frac{\boldsymbol{\rho}_{1}}{D}}\left[p_{0}\left(1-u^{2}\right)+n_{0} u^{2}-\boldsymbol{\rho}_{3} c_{1}^{2}\right], \\
& m_{3}=-\sqrt{\frac{\boldsymbol{\rho}_{3}}{D}}\left(q_{0}+p_{0}\right) u \sqrt{1-u^{2}}
\end{aligned}
$$

with Also, the wave speeds are

$D=\boldsymbol{\rho}_{1}\left[p_{0}+\left(n_{0}-p_{0}\right) u^{2}-\boldsymbol{\rho}_{3} c_{1}^{2}\right]^{2}+\boldsymbol{\rho}_{3}\left(q_{0}+p_{0}\right)^{2}\left(1-u^{2}\right) u^{2}$.

$$
c_{1,3}^{2}=\frac{1}{2}\left\{\begin{array}{c}
\frac{k_{0}+m_{0}}{\rho_{1}}+\frac{p_{0}}{\rho_{3}}+\left(\frac{p_{0}-k_{0}-m_{0}}{\rho_{1}}+\frac{n_{0}-p_{0}}{\rho_{3}}\right) u^{2} \\
\pm\left\{\frac{k_{0}+m_{0}}{\rho_{1}}-\frac{p_{0}}{\rho_{3}}-\left(\frac{k_{0}+m_{0}-p_{0}}{\rho_{1}}+\frac{n_{0}-p_{0}}{\rho_{3}}\right) u^{2}\right]^{2} \\
+4 \frac{\left(l_{0}+p_{0}\right)\left(q_{0}+p_{0}\right)}{\rho_{1} \rho_{3}}\left(1-u^{2}\right) u^{2}
\end{array}\right\},
$$

Then,

$$
\overline{\mathbf{S}}_{\mathbf{x}}\left(\mathbf{L}_{1}-\overline{\mathbf{L}}\right)=\left(\begin{array}{c}
4\left(k_{1}-\bar{k}\right) k_{s}+2\left(q_{1}-\bar{q}\right) l_{s}, 2\left(l_{1}-\bar{l}\right) k_{s}+\left(n_{1}-\bar{n}\right) l_{s}, \\
2\left(k_{1}-\bar{k}\right) q_{s}+\left(q_{1}-\bar{q}\right) n_{s}, 2\left(l_{1}-\bar{l}\right) q_{s}+\left(n_{1}-\bar{n}\right) n_{s} \\
4\left(m_{1}-\bar{m}\right) m_{s}, 4\left(p_{1}-\bar{p}\right) p_{s}
\end{array}\right)
$$


At the same time, let

$$
\mathbf{I}=(1,0,0,1,1,1) .
$$

$$
\begin{gathered}
\mathbf{I}+\overline{\mathbf{S}}_{\mathbf{x}}\left(\mathbf{L}_{1}-\overline{\mathbf{L}}\right)=\left(\begin{array}{c}
4\left(k_{1}-\bar{k}\right) k_{s}+2\left(q_{1}-\bar{q}\right) l_{s}+1,2\left(l_{1}-\bar{l}\right) k_{s}+\left(n_{1}-\bar{n}\right) l_{s}, \\
2\left(k_{1}-\bar{k}\right) q_{s}+\left(q_{1}-\bar{q}\right) n_{s}, 2\left(l_{1}-\bar{l}\right) q_{s}+\left(n_{1}-\bar{n}\right) n_{s}+1, \\
4\left(m_{1}-\bar{m}\right) m_{s}+1,4\left(p_{1}-\bar{p}\right) p_{s}+1
\end{array}\right), \\
{\left[\mathbf{I}+\overline{\mathbf{S}}_{\mathbf{x}}\left(\mathbf{L}_{1}-\overline{\mathbf{L}}\right)\right]^{-1}=\left(\begin{array}{c}
\frac{2\left(l_{1}-\bar{l}\right) q_{s}+\left(n_{1}-\bar{n}\right) n_{s}+1}{2 \lambda},-\frac{2\left(l_{1}-\bar{l}\right) k_{s}+\left(n_{1}-\bar{n}\right) l_{s}}{2 \lambda} \\
-\frac{2\left(k_{1}-\bar{k}\right) q_{s}+\left(q_{1}-\bar{q}\right) n_{s}}{2 \lambda}, \frac{4\left(k_{1}-\bar{k}\right) k_{s}+2\left(q_{1}-\bar{q}\right) l_{s}+1}{2 \lambda} \\
\frac{1}{4\left(m_{1}-\bar{m}\right) m_{s}+1}, \frac{1}{4\left(p_{1}-\bar{p}\right) p_{s}+1}
\end{array}\right),}
\end{gathered}
$$

where

$$
\begin{aligned}
\lambda= & {\left[2\left(k_{1}-\bar{k}\right) k_{s}+\left(q_{1}-\bar{q}\right) l_{s}+\frac{1}{2}\right]\left[2\left(l_{1}-\bar{l}\right) q_{s}+\left(n_{1}-\bar{n}\right) n_{s}+1\right] } \\
& -\left[2\left(l_{1}-\bar{l}\right) k_{s}+\left(n_{1}-\bar{n}\right) l_{s}\right]\left[2\left(k_{1}-\bar{k}\right) q_{s}+\left(q_{1}-\bar{q}\right) n_{s}\right] .
\end{aligned}
$$

Then,

$$
\begin{aligned}
& \left(\mathbf{L}_{1}-\overline{\mathbf{L}}\right)\left[\mathbf{I}+\overline{\mathbf{S}}_{\mathbf{x}}\left(\mathbf{L}_{1}-\overline{\mathbf{L}}\right)\right]^{-1} \\
& \quad\left(\begin{array}{c}
\frac{\left(k_{1}-\bar{k}\right)\left[2\left(l_{1}-\bar{l}\right) q_{s}+\left(n_{1}-\bar{n}\right) n_{s}+1\right]-\left(l_{1}-\bar{l}\right)\left[2\left(l_{1}-\bar{l}\right) k_{s}+\left(n_{1}-\bar{n}\right) l_{s}\right]}{\lambda}, \\
\frac{-\left(k_{1}-\bar{k}\right)\left[2\left(l_{1}-\bar{l}\right) k_{s}+\left(n_{1}-\bar{n}\right) l_{s}\right]+\left(l_{1}-\bar{l}\right)\left[2\left(k_{1}-\bar{k}\right) k_{s}+\left(q_{1}-\bar{q}\right) l_{s}+(1 / 2)\right]}{\lambda}, \\
\frac{-\left(q_{1}-\bar{q}\right)\left[2\left(l_{1}-\bar{l}\right) k_{s}+\left(n_{1}-\bar{n}\right) l_{s}\right]+\left(n_{1}-\bar{n}\right)\left[2\left(k_{1}-\bar{k}\right) k_{s}+\left(q_{1}-\bar{q}\right) l_{s}+(1 / 2)\right]}{\lambda}, \\
\frac{\left.2\left(m_{1}-\bar{m}\right) q_{s}+\left(n_{1}-\bar{n}\right) n_{s}+1\right]-\left(n_{1}-\bar{n}\right)\left[2\left(k_{1}-\bar{k}\right) q_{s}+\left(q_{1}-\bar{q}\right) n_{s}\right]}{2 \lambda}, \\
\frac{2\left(m_{1}-\bar{m}\right) m_{s}+1}{4\left(p_{1}-\bar{p}\right) p_{s}+1}
\end{array}\right.
\end{aligned}
$$


Similarly, for density, let the inclusion density $\boldsymbol{\rho}_{1}=\left(\boldsymbol{\rho}_{1}, \boldsymbol{\rho}_{3}\right)$, the dynamic density $\overline{\boldsymbol{\rho}}=\left(\overline{\boldsymbol{\rho}}_{1}, \overline{\boldsymbol{\rho}}_{3}\right)$, and $\overline{\mathbf{M}}_{t}=\left(\mathbf{M}_{1}, \mathbf{M}_{3}\right)$, then,

$$
\left[\mathbf{I}+\overline{\mathbf{M}}_{t}\left(\boldsymbol{\rho}_{1}-\overline{\boldsymbol{\rho}}\right)\right]^{-1}=\left(\frac{1}{1+\mathbf{M}_{1}\left(\boldsymbol{\rho}_{1}-\overline{\boldsymbol{\rho}}_{1}\right)}, \frac{1}{1+\mathbf{M}_{3}\left(\boldsymbol{\rho}_{3}-\overline{\boldsymbol{\rho}}_{3}\right)}\right),
$$

where the material parameters of $\overline{\mathbf{S}}_{\mathbf{x}}$ and $\overline{\mathbf{M}}_{t}$ are all functions of inclusion volume fraction with the differential method which are dynamic effective properties. The specific forms of the terms in $\overline{\mathbf{M}}_{t}$ are

$$
\begin{aligned}
& \mathbf{M}_{1}=\frac{1}{2 \boldsymbol{\rho}_{1}} \int_{0}^{1} \mathrm{~d} u\left[m_{1}^{2} F_{1}(\zeta(u))+F_{2}(\zeta(u))+m_{3}^{2} F_{3}(\zeta(u))\right], \\
& \mathbf{M}_{3}=\frac{1}{\boldsymbol{\rho}_{3}} \int_{0}^{1} \mathrm{~d} u\left[m_{3}^{2} F_{1}(\zeta(u))+m_{1}^{2} F_{3}(\zeta(u))\right] .
\end{aligned}
$$

Equations (41) and (42) can be rewritten from equations (B.20) and (B.21) as follows:

$$
\begin{aligned}
& \int \frac{\mathrm{d} \bar{k}}{\mathrm{~d} f_{1}}=\frac{1}{2} \frac{h(k \mathbf{n}) h(k \mathbf{n})}{1-f_{1}} \\
& \times \frac{\left(k_{1}-\bar{k}\right)\left[2\left(l_{1}-\bar{l}\right) q_{s}+\left(n_{1}-\bar{n}\right) n_{s}+1\right]-\left(l_{1}-\bar{l}\right)\left[2\left(l_{1}-\bar{l}\right) k_{s}+\left(n_{1}-\bar{n}\right) l_{s}\right]}{\lambda}, \\
& \frac{\mathrm{d} \bar{l}}{\mathrm{~d} f_{1}}=\frac{h(k \mathbf{n}) h(k \mathbf{n})}{1-f_{1}} \\
& \times \frac{-\left(k_{1}-\bar{k}\right)\left[2\left(l_{1}-\bar{l}\right) k_{s}+\left(n_{1}-\bar{n}\right) l_{s}\right]+\left(l_{1}-\bar{l}\right)\left[2\left(k_{1}-\bar{k}\right) k_{s}+\left(q_{1}-\bar{q}\right) l_{s}+1 / 2\right]}{\lambda} \\
& \frac{\mathrm{d} \bar{q}}{\mathrm{~d} f_{1}}=\frac{h(k \mathbf{n}) h(k \mathbf{n})}{1-f_{1}} \\
& \times \frac{\left(q_{1}-\bar{q}\right)\left[2\left(l_{1}-\bar{l}\right) q_{s}+\left(n_{1}-\bar{n}\right) n_{s}+1\right]-\left(n_{1}-\bar{n}\right)\left[2\left(k_{1}-\bar{k}\right) q_{s}+\left(q_{1}-\bar{q}\right) n_{s}\right]}{2 \lambda}, \\
& \frac{\mathrm{d} \bar{n}}{\mathrm{~d} f_{1}}=\frac{h(k \mathbf{n}) h(k \mathbf{n})}{1-f_{1}} \\
& \times \frac{-\left(q_{1}-\bar{q}\right)\left[2\left(l_{1}-\bar{l}\right) k_{s}+\left(n_{1}-\bar{n}\right) l_{s}\right]+\left(n_{1}-\bar{n}\right)\left[2\left(k_{1}-\bar{k}\right) k_{s}+\left(q_{1}-\bar{q}\right) l_{s}+1 / 2\right]}{\lambda}, \\
& \frac{\mathrm{d} \bar{m}}{\mathrm{~d} f_{1}}=\frac{h(k \mathbf{n}) h(k \mathbf{n})}{1-f_{1}} \frac{\left(m_{1}-\bar{m}\right)}{4\left(m_{1}-\bar{m}\right) m_{s}+1} \\
& \frac{\mathrm{d} \bar{p}}{\mathrm{~d} f_{1}}=\frac{h(k \mathbf{n}) h(k \mathbf{n})}{1-f_{1}} \frac{\left(p_{1}-\bar{p}\right)}{4\left(p_{1}-\bar{p}\right) p_{s}+1} \\
& \frac{\mathrm{d} \overline{\boldsymbol{\rho}}_{1}}{\mathrm{~d} f_{1}}=\frac{h(k \mathbf{n}) h(k \mathbf{n})}{1-f_{1}} \frac{\left(\boldsymbol{\rho}_{1}-\overline{\boldsymbol{\rho}}_{1}\right)}{1+\mathbf{M}_{1}\left(\boldsymbol{\rho}_{1}-\overline{\boldsymbol{\rho}}_{1}\right)}, \\
& \frac{\mathrm{d} \overline{\boldsymbol{\rho}}_{3}}{\mathrm{~d} f_{1}}=\frac{h(k \mathbf{n}) h(k \mathbf{n})}{1-f_{1}} \frac{\left(\boldsymbol{\rho}_{3}-\overline{\boldsymbol{\rho}}_{3}\right)}{1+\mathbf{M}_{3}\left(\boldsymbol{\rho}_{3}-\overline{\boldsymbol{\rho}}_{3}\right)}
\end{aligned}
$$

The corresponding initial conditions are 


$$
\left\{\begin{array}{l}
\left.\bar{k}\left(f_{1}\right)\right|_{f_{1}=0}=k_{2}, \\
\left.\bar{l}\left(f_{1}\right)\right|_{f_{1}=0}=l_{2}, \\
\left.\bar{q}\left(f_{1}\right)\right|_{f_{1}=0}=q_{2}, \\
\left.\bar{n}\left(f_{1}\right)\right|_{f_{1}=0}=n_{2}, \\
\left.\bar{m}\left(f_{1}\right)\right|_{f_{1}=0}=m_{2}, \\
\left.\bar{p}\left(f_{1}\right)\right|_{f_{1}=0}=p_{2}, \\
\left.\overline{\boldsymbol{\rho}}_{1}\left(f_{1}\right)\right|_{f_{1}=0}=\left(\boldsymbol{\rho}_{1}\right)_{2}, \\
\left.\overline{\boldsymbol{\rho}}_{3}\left(f_{1}\right)\right|_{f_{1}=0}=\left(\boldsymbol{\rho}_{3}\right)_{2} .
\end{array}\right.
$$

Equations (B.24) and (B.25) are numerically solved by the fourth-order Runge-Kutta method, and the iterative steps are as follows:

$$
y_{p, n+1}=y_{p, n}+\frac{h}{6}\left(K_{p, 1}+2 K_{p, 2}+2 K_{p, 3}+K_{p, 4}\right)
$$

where $y_{p, n}$ is the $n$-th item of $y_{p}$, such as $y_{p, 0}$ is the initial condition of $y_{p}$ when $f_{1}=0, h$ is the step length, and

$$
\left\{\begin{array}{l}
K_{p, 1}=g_{p}\left(\left(f_{1}\right)_{n}, y_{1}, y_{2}, \cdots y_{8}\right), \\
K_{p, 2}=g_{p}\left(\left(f_{1}\right)_{n}+\frac{h}{2}, y_{1}+\frac{h}{2} K_{1,1}, y_{2}+\frac{h}{2} K_{2,1}, \cdots, y_{8}+\frac{h}{2} K_{8,1}\right), \\
K_{p, 3}=g_{p}\left(\left(f_{1}\right)_{n}+\frac{h}{2}, y_{1}+\frac{h}{2} K_{1,2}, y_{2}+\frac{h}{2} K_{2,2}, \cdots, y_{8}+\frac{h}{2} K_{8,2}\right), \\
K_{p, 4}=g_{p}\left(\left(f_{1}\right)_{n}+h, y_{1}+h K_{1,3}, y_{2}+h K_{2,3}, \cdots, y_{8}+h K_{8,3}\right), \\
p=1,2,3 \cdots, 8, \\
\qquad \begin{array}{l}
y_{1}=\bar{k}\left(f_{1}\right), \\
y_{2}=\bar{l}\left(f_{1}\right), \\
y_{3}=\bar{q}\left(f_{1}\right), \\
y_{4}=\bar{n}\left(f_{1}\right), \\
y_{5}=\bar{m}\left(f_{1}\right), \\
y_{6}=\bar{p}\left(f_{1}\right), \\
y_{7}=\overline{\boldsymbol{\rho}}\left(f_{1}\right), \\
y_{8}=\overline{\boldsymbol{\rho}}\left(f_{1}\right) .
\end{array}
\end{array}\right.
$$

Specific iterations are as follows: when $f_{1}=0$, namely $n=0$, we have

$$
\left\{\begin{array}{l}
K_{1,1}=\frac{1}{2} \frac{h(k \mathbf{n}) h(k \mathbf{n})}{1-f_{1}} \\
\times \frac{\left(k_{1}-y_{1}\right)\left[2\left(l_{1}-y_{2}\right) q_{s}+\left(n_{1}-y_{4}\right) n_{s}+1\right]-\left(l_{1}-y_{2}\right)\left[2\left(l_{1}-y_{2}\right) k_{s}+\left(n_{1}-y_{4}\right) l_{s}\right]}{\lambda}, \\
K_{2,1}=\frac{h(k \mathbf{n}) h(k \mathbf{n})}{1-f_{1}} \\
\times \frac{-\left(k_{1}-y_{1}\right)\left[2\left(l_{1}-y_{2}\right) k_{s}+\left(n_{1}-y_{4}\right) l_{s}\right]+\left(l_{1}-y_{2}\right)\left[2\left(k_{1}-y_{1}\right) k_{s}+\left(q_{1}-y_{3}\right) l_{s}+1 / 2\right]}{\lambda} \\
K_{3,1}=\frac{h(k \mathbf{n}) h(k \mathbf{n})}{1-f_{1}} \\
\times \frac{\left(q_{1}-y_{3}\right)\left[2\left(l_{1}-y_{2}\right) q_{s}+\left(n_{1}-y_{4}\right) n_{s}+1\right]-\left(n_{1}-y_{4}\right)\left[2\left(k_{1}-y_{1}\right) q_{s}+\left(q_{1}-y_{3}\right) n_{s}\right]}{2 \lambda} \\
K_{4,1}=\frac{h(k \mathbf{n}) h(k \mathbf{n})}{1-f_{1}} \\
K_{7,1}=\frac{h(k \mathbf{n}) h(k \mathbf{n})}{1-f_{1}} \frac{\left(\boldsymbol{\rho}_{1}-y_{7}\right)}{1+\mathbf{M}_{1}\left(\boldsymbol{\rho}_{1}-y_{7}\right)}, \\
K_{8,1}=\frac{h(k \mathbf{n}) h(k \mathbf{n})}{1-f_{1}} \frac{\left(p_{1}-y_{6}\right)}{4\left(p_{1}-y_{6}\right) p_{s}+1}, \\
K_{5,1}=\frac{h(k \mathbf{n}) h(k \mathbf{n})}{1-f_{1}} \frac{\left(m_{1}-y_{5}\right)}{4\left(m_{1}-y_{5}\right) m_{s}+1},
\end{array}\right.
$$




$$
\begin{aligned}
& K_{1,2}=\frac{1}{2} \frac{h(k \mathbf{n}) h(k \mathbf{n})}{1-\left(f_{1}+h / 2\right)} \times \frac{1}{\lambda} \\
& \times\left(\begin{array}{c}
\left(k_{1}-\left(y_{1}+\frac{h}{2} K_{1,1}\right)\right)\left[2\left(l_{1}-\left(y_{2}+\frac{h}{2} K_{2,1}\right)\right) q_{s}+\left(n_{1}-\left(y_{4}+\frac{h}{2} K_{4,1}\right)\right) n_{s}+1\right] \\
-\left(l_{1}-\left(y_{2}+\frac{h}{2} K_{2,1}\right)\right)\left[2\left(l_{1}-\left(y_{2}+\frac{h}{2} K_{2,1}\right)\right) k_{s}+\left(n_{1}-\left(y_{4}+\frac{h}{2} K_{4,1}\right)\right) l_{s}\right]
\end{array}\right), \\
& K_{2,2}=\frac{h(k \mathbf{n}) h(k \mathbf{n})}{1-\left(f_{1}+h / 2\right)_{1}} \times \frac{1}{\lambda} \\
& \times\left(\begin{array}{c}
-\left(k_{1}-\left(y_{1}+\frac{h}{2} K_{1,1}\right)\right)\left[2\left(l_{1}-\left(y_{2}+\frac{h}{2} K_{2,1}\right)\right) k_{s}+\left(n_{1}-\left(y_{4}+\frac{h}{2} K_{4,1}\right)\right) l_{s}\right] \\
+\left(l_{1}-\left(y_{2}+\frac{h}{2} K_{2,1}\right)\right)\left[2\left(k_{1}-\left(y_{1}+\frac{h}{2} K_{1,1}\right)\right) k_{s}+\left(q_{1}-\left(y_{3}+\frac{h}{2} K_{3,1}\right)\right) l_{s}+\frac{1}{2}\right]
\end{array}\right), \\
& K_{3,2}=\frac{h(k \mathbf{n}) h(k \mathbf{n})}{1-\left(f_{1}+h / 2\right)_{1}} \times \frac{1}{2 \lambda} \\
& \times\left(\begin{array}{l}
\left(q_{1}-\left(y_{3}+\frac{h}{2} K_{3,1}\right)\right)\left[2\left(l_{1}-\left(y_{2}+\frac{h}{2} K_{2,1}\right)\right) q_{s}+\left(n_{1}-\left(y_{4}+\frac{h}{2} K_{4,1}\right)\right) n_{s}+1\right] \\
-\left(n_{1}-\left(y_{4}+\frac{h}{2} K_{4,1}\right)\right)\left[2\left(k_{1}-\left(y_{1}+\frac{h}{2} K_{1,1}\right)\right) q_{s}+\left(q_{1}-\left(y_{3}+\frac{h}{2} K_{3,1}\right)\right) n_{s}\right]
\end{array}\right), \\
& K_{4,2}=\frac{h(k \mathbf{n}) h(k \mathbf{n})}{1-\left(f_{1}+h / 2\right)} \times \frac{1}{\lambda} \\
& \times\left(\begin{array}{c}
-\left(q_{1}-\left(y_{3}+\frac{h}{2} K_{3,1}\right)\right)\left[2\left(l_{1}-\left(y_{2}+\frac{h}{2} K_{2,1}\right)\right) k_{s}+\left(n_{1}-\left(y_{4}+\frac{h}{2} K_{4,1}\right)\right) l_{s}\right] \\
+\left(n_{1}-\left(y_{4}+\frac{h}{2} K_{4,1}\right)\right)\left[2\left(k_{1}-\left(y_{1}+\frac{h}{2} K_{1,1}\right)\right) k_{s}+\left(q_{1}-\left(y_{3}+\frac{h}{2} K_{3,1}\right)\right) l_{s}+\frac{1}{2}\right]
\end{array}\right), \\
& K_{5,2}=\frac{h(k \mathbf{n}) h(k \mathbf{n})}{1-\left(f_{1}+h / 2\right)} \frac{\left(m_{1}-\left(y_{5}+(h / 2) K_{5,1}\right)\right)}{4\left(m_{1}-\left(y_{5}+(h / 2) K_{5,1}\right)\right) m_{s}+1} \\
& K_{6,2}=\frac{h(k \mathbf{n}) h(k \mathbf{n})}{1-\left(f_{1}+h / 2\right)} \frac{\left(p_{1}-\left(y_{6}+(h / 2) K_{6,1}\right)\right)}{4\left(p_{1}-\left(y_{6}+(h / 2) K_{6,1}\right)\right) p_{s}+1} \\
& K_{7,2}=\frac{h(k \mathbf{n}) h(k \mathbf{n})}{1-\left(f_{1}+h / 2\right)} \frac{\left(\boldsymbol{\rho}_{1}-\left(y_{7}+(h / 2) K_{7,1}\right)\right)}{1+\mathbf{M}_{1}\left(\boldsymbol{\rho}_{1}-\left(y_{7}+(h / 2) K_{7,1}\right)\right)}, \\
& K_{8,2}=\frac{h(k \mathbf{n}) h(k \mathbf{n})}{1-\left(f_{1}+h / 2\right)} \frac{\left(\boldsymbol{\rho}_{3}-\left(y_{8}+(h / 2) K_{8,1}\right)\right)}{1+\mathbf{M}_{3}\left(\boldsymbol{\rho}_{3}-\left(y_{8}+(h / 2) K_{8,1}\right)\right)},
\end{aligned}
$$




$$
\begin{aligned}
& K_{1,3}=\frac{1}{2} \frac{h(k \mathbf{n}) h(k \mathbf{n})}{1-\left(f_{1}+h / 2\right)} \times \frac{1}{\lambda} \\
& \times\left(\begin{array}{c}
\left(k_{1}-\left(y_{1}+\frac{h}{2} K_{1,2}\right)\right)\left[2\left(l_{1}-\left(y_{2}+\frac{h}{2} K_{2,2}\right)\right) q_{s}+\left(n_{1}-\left(y_{4}+\frac{h}{2} K_{4,2}\right)\right) n_{s}+1\right] \\
-\left(l_{1}-\left(y_{2}+\frac{h}{2} K_{2,2}\right)\right)\left[2\left(l_{1}-\left(y_{2}+\frac{h}{2} K_{2,2}\right)\right) k_{s}+\left(n_{1}-\left(y_{4}+\frac{h}{2} K_{4,2}\right)\right) l_{s}\right]
\end{array}\right), \\
& K_{2,3}=\frac{h(k \mathbf{n}) h(k \mathbf{n})}{1-\left(f_{1}+h / 2\right)_{1}} \times \frac{1}{\lambda} \\
& \times\left(\begin{array}{c}
-\left(k_{1}-\left(y_{1}+\frac{h}{2} K_{1,2}\right)\right)\left[2\left(l_{1}-\left(y_{2}+\frac{h}{2} K_{2,2}\right)\right) k_{s}+\left(n_{1}-\left(y_{4}+\frac{h}{2} K_{4,2}\right)\right) l_{s}\right] \\
+\left(l_{1}-\left(y_{2}+\frac{h}{2} K_{2,2}\right)\right)\left[2\left(k_{1}-\left(y_{1}+\frac{h}{2} K_{1,2}\right)\right) k_{s}+\left(q_{1}-\left(y_{3}+\frac{h}{2} K_{3,2}\right)\right) l_{s}+\frac{1}{2}\right]
\end{array}\right), \\
& K_{3,3}=\frac{h(k \mathbf{n}) h(k \mathbf{n})}{1-\left(f_{1}+h / 2\right)_{1}} \times \frac{1}{2 \lambda} \\
& \times\left(\begin{array}{c}
\left(q_{1}-\left(y_{3}+\frac{h}{2} K_{3,2}\right)\right)\left[2\left(l_{1}-\left(y_{2}+\frac{h}{2} K_{2,2}\right)\right) q_{s}+\left(n_{1}-\left(y_{4}+\frac{h}{2} K_{4,2}\right)\right) n_{s}+1\right] \\
-\left(n_{1}-\left(y_{4}+\frac{h}{2} K_{4,2}\right)\right)\left[2\left(k_{1}-\left(y_{1}+\frac{h}{2} K_{1,2}\right)\right) q_{s}+\left(q_{1}-\left(y_{3}+\frac{h}{2} K_{3,2}\right)\right) n_{s}\right]
\end{array}\right), \\
& K_{4,3}=\frac{h(k \mathbf{n}) h(k \mathbf{n})}{1-\left(f_{1}+h / 2\right)} \times \frac{1}{\lambda} \\
& \times\left(\begin{array}{c}
-\left(q_{1}-\left(y_{3}+\frac{h}{2} K_{3,2}\right)\right)\left[2\left(l_{1}-\left(y_{2}+\frac{h}{2} K_{2,2}\right)\right) k_{s}+\left(n_{1}-\left(y_{4}+\frac{h}{2} K_{4,2}\right)\right) l_{s}\right] \\
+\left(n_{1}-\left(y_{4}+\frac{h}{2} K_{4,2}\right)\right)\left[2\left(k_{1}-\left(y_{1}+\frac{h}{2} K_{1,2}\right)\right) k_{s}+\left(q_{1}-\left(y_{3}+\frac{h}{2} K_{3,2}\right)\right) l_{s}+\frac{1}{2}\right]
\end{array}\right), \\
& K_{5,3}=\frac{h(k \mathbf{n}) h(k \mathbf{n})}{1-\left(f_{1}+h / 2\right)} \frac{\left(m_{1}-\left(y_{5}+(h / 2) K_{5,2}\right)\right)}{4\left(m_{1}-\left(y_{5}+(h / 2) K_{5,2}\right)\right) m_{s}+1}, \\
& K_{6,3}=\frac{h(k \mathbf{n}) h(k \mathbf{n})}{1-\left(f_{1}+h / 2\right)} \frac{\left(p_{1}-\left(y_{6}+(h / 2) K_{6,2}\right)\right)}{4\left(p_{1}-\left(y_{6}+(h / 2) K_{6,2}\right)\right) p_{s}+1}, \\
& K_{7,3}=\frac{h(k \mathbf{n}) h(k \mathbf{n})}{1-\left(f_{1}+h / 2\right)} \frac{\left(\boldsymbol{\rho}_{1}-\left(y_{7}+(h / 2) K_{7,2}\right)\right)}{1+\mathbf{M}_{1}\left(\boldsymbol{\rho}_{1}-\left(y_{7}+(h / 2) K_{7,2}\right)\right)}, \\
& K_{8,3}=\frac{h(k \mathbf{n}) h(k \mathbf{n})}{1-\left(f_{1}+h / 2\right)} \frac{\left(\boldsymbol{\rho}_{3}-\left(y_{8}+(h / 2) K_{8,2}\right)\right)}{1+\mathbf{M}_{3}\left(\boldsymbol{\rho}_{3}-\left(y_{8}+(h / 2) K_{8,2}\right)\right)},
\end{aligned}
$$


Advances in Materials Science and Engineering

19

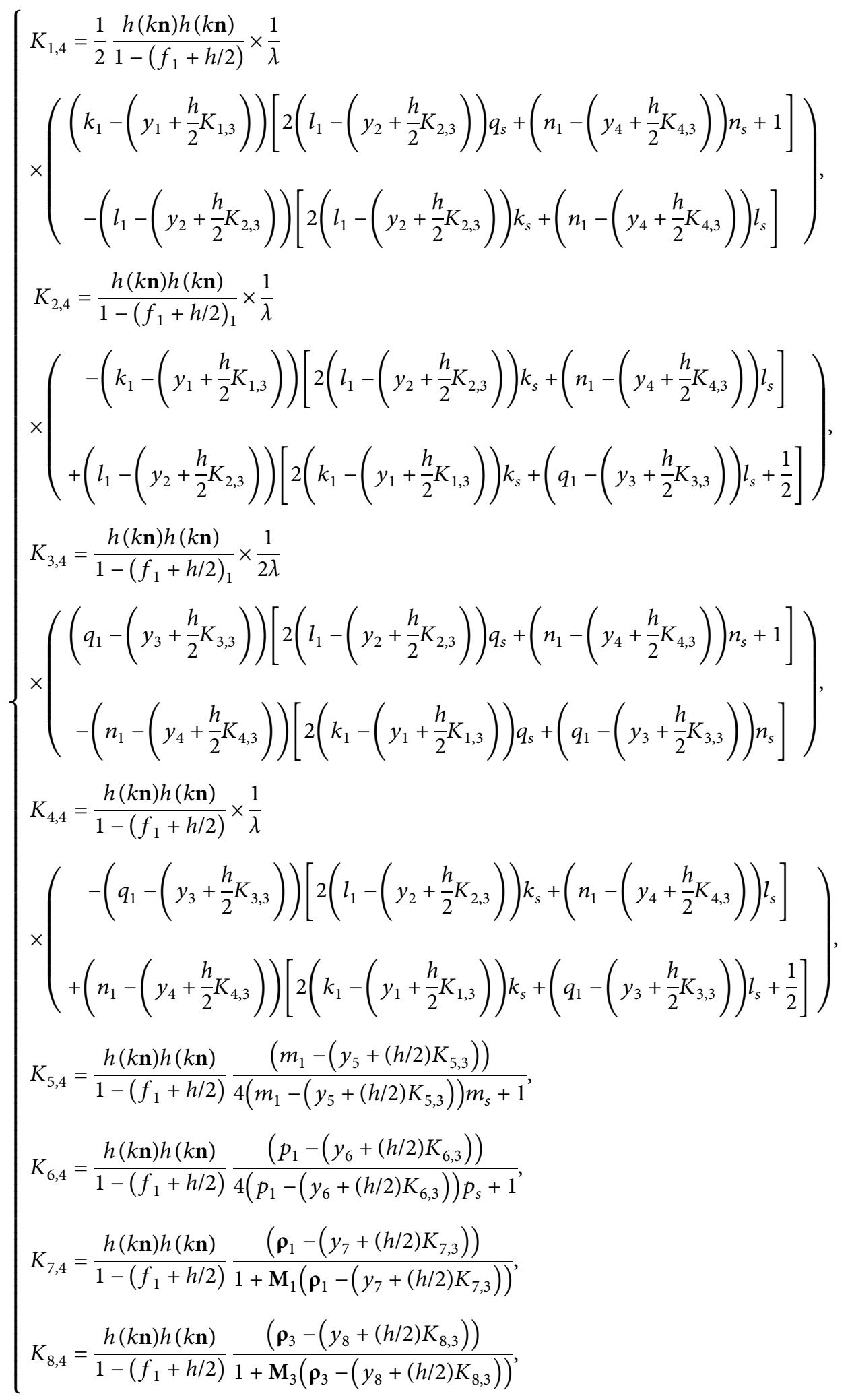


where $y_{p}(p=1,2,3, \ldots, 8)$ are initial conditions and equal to $y_{p, 0}(p=1,2,3, \ldots, 8)$.

According to equation (B.26), we obtain $y_{p, 1}(p=1,2,3, \ldots, 8)$, then, let $f_{1}=h, f_{1}=2 h, \ldots$, and by repeating the steps of equations (B.29) (B.32), we obtain $y_{p, 2}, y_{p, 3}, \ldots$, if the inclusion volume fraction $f_{1}$ equals to the value required, then, we obtain the results we need.

\section{Conflicts of Interest}

The authors declare that they have no conflicts of interest.

\section{Acknowledgments}

This work was supported by the National Natural Science Foundation of China (project no. 12072240).

\section{References}

[1] M. Saiyad, N. M. Devashrayee, and R. K. Mevada, "Study the effect of dispersion of filler in polymer composite for radiation shielding," Polymer Composites, vol. 35, no. 7, pp. 1263-1266, 2014.

[2] K. Nakamura, T. Ueda, S. Hosono, and T. Maruno, "Theoretical analysis of the decay of shear strength of adhesion in metal/epoxy/metal joints in an aqueous environment," International Journal of Adhesion and Adhesives, vol. 7, no. 4, pp. 209-212, 1987.

[3] A. H. Ali, "Study the enhancement of the radiation shielding afforded to epoxy/lead composites," J. of university of Anbar for pure science, vol. 7, no. 2, 2013.

[4] T. R. Tauchert and A. N. Guzelsu, "An experimental study of dispersion of stress waves in a fiber-reinforced composite," Journal of Applied Mechanics, vol. 39, no. 1, pp. 98-102, 1972.

[5] V. K. Kinra, "Dispersive wave propagation in random particulate composites," in Recent Advances in Composites in the United States and JapanASTM International, West Conshohocken, PA, USA, 1985.

[6] L. Wang and F. Yuan, "Experimental study of Lamb wave propagation in composite laminates," in Smart Structures and Materials 2006: Sensors and Smart Structures Technologies for Civil, Mechanical, and Aerospace Systemsvol. 6174, , pp. 1301-1312, International Society for Optics and Photonics, 2006.

[7] J. R. Willis, "Exact effective relations for dynamics of a laminated body," Mechanics of Materials, vol. 41, no. 4, pp. 385-393, 2009.

[8] S. Nemat-Nasser and A. Srivastava, "Overall dynamic constitutive relations of layered elastic composites," Journal of the Mechanics and Physics of Solids, vol. 59, no. 10, pp. 1953-1965, 2011.

[9] A. Srivastava and S. Nemat-Nasser, "Overall dynamic properties of three-dimensional periodic elastic composites," Proceedings of the Royal Society A: Mathematical, Physical \& Engineering Sciences, vol. 468, no. 2137, pp. 269-287, 2011.

[10] M. M. Sigalas and C. M. Soukoulis, "Elastic-wave propagation through disordered and/or absorptive layered systems," Physical Review B, vol. 51, no. 5, pp. 2780-2789, 1995.

[11] Z. Hou, X. Fu, and Y. Liu, "Calculational method to study the transmission properties of phononic crystals," Physical Review $B$, vol. 70, no. 1, 2004.
[12] Z. Hou, W. Kuang, and Y. Liu, "Transmission property analysis of a two-dimensional phononic crystal," Physics Letters A, vol. 333, no. 1-2, pp. 172-180, 2004.

[13] M. M. Sigalas and E. N. Economou, "Elastic and acoustic wave band structure," Journal of Sound and Vibration, vol. 158, no. 2, pp. 377-382, 1992.

[14] Z. Yan and Y. Wang, "Wavelet-based method for computing elastic band gaps of one-dimensional phononic crystals," Science in China - Series G: Physics Mechanics and Astronomy, vol. 50, no. 5, pp. 622-630, 2007.

[15] D. García-Pablos, M. Sigalas, F. R. Montero de Espinosa, M. Torres, M. Kafesaki, and N. García, "Theory and experiments on elastic band gaps," Physical Review Letters, vol. 84, no. 19, pp. 4349-4352, 2000.

[16] M. Kafesaki and E. N. Economou, "Multiple-scattering theory for three-dimensional periodic acoustic composites," Physical Review B, vol. 60, no. 17, pp. 11993-12001, 1999.

[17] W. Kohn, J. A. Krumhansl, and E. H. Lee, "Variational methods for dispersion relations and elastic properties of composite materials," Journal of Applied Mechanics, vol. 39, no. 2, pp. 317-318, 1972.

[18] M. I. Hussein, "Reduced Bloch mode expansion for periodic media band structure calculations," Proceedings of the Royal Society A: Mathematical, Physical \& Engineering Sciences, vol. 465, no. 2109, pp. 2825-2848, 2009.

[19] S. Tsinopoulos, J. Verbis, and D. Polyzos, "An iterative effective medium approximation for wave dispersion and attenuation predictions in particulate composites," Advanced Composites Letters, vol. 9, no. 3, 2000.

[20] J. T. Verbis, S. E. Kattis, S. V. Tsinopoulos, and D. Polyzos, "Wave dispersion and attenuation in fiber composites," Computational Mechanics, vol. 27, no. 3, pp. 244-252, 2001.

[21] S. K. Kanaun and V. M. Levin, "Effective medium method in the problem of axial elastic shear wave propagation through fiber composites," International Journal of Solids and Structures, vol. 40, no. 18, pp. 4859-4878, 2003.

[22] J. Y. Kim, J. G. Ih, and B. H. Lee, "Dispersion of elastic waves in random particulate composites," Journal of the Acoustical Society of America, vol. 97, no. 3, pp. 1380-1388, 1995.

[23] F. J. Sabina and J. R. Willis, "A simple self-consistent analysis of wave propagation in particulate composites," Wave Motion, vol. 10, no. 2, pp. 127-142, 1988.

[24] F. Sabina, V. Smyshlyaev, and J. Willis, "Self-consistent analysis of waves in a matrix-inclusion composite-I. Aligned spheroidal inclusions," Journal of the Mechanics and Physics of Solids, vol. 41, no. 10, pp. 1573-1588, 1993.

[25] F. J. Sabina, C. A. Gandarrilla-Pérez, J. A. Otero et al., "Dynamic homogenization for composites with embedded multioriented ellipsoidal inclusions," International Journal of Solids and Structures, vol. 69-70, pp. 121-130, 2015.

[26] P. G. J. Bussink, P. L. Iske, J. Oortwijn, and G. L. M. M. Verbist, "Self-consistent analysis of elastic wave propagation in two-dimensional matrix-inclusion composites," Journal of the Mechanics and Physics of Solids, vol. 43, no. 10, pp. 1673-1690, 1995.

[27] R. W. Zimmerman, "Elastic moduli of a solid containing spherical inclusions," Mechanics of Materials, vol. 12, no. 1, pp. 17-24, 1991.

[28] N. Ramakrishnan and V. S. Arunachalam, "Effective elastic moduli of porous ceramic materials," Journal of the American Ceramic Society, vol. 76, no. 11, pp. 2745-2752, 1993.

[29] A. D. Drozdov and J. Claville Christiansen, "The effect of porosity on elastic moduli of polymer foams," Journal of 
Applied Polymer Science, vol. 137, no. 10, Article ID 48449, 2020.

[30] J. Willis, "Polarization approach to the scattering of elastic waves-I. Scattering by a single inclusion," Journal of the Mechanics and Physics of Solids, vol. 28, no. 5-6, pp. 287-305, 1980.

[31] A. N. Norris, A. J. Callegari, and P. Sheng, "A generalized differential effective medium theory," Journal of the Mechanics and Physics of Solids, vol. 33, no. 6, pp. 525-543, 1985.

[32] A. N. Norris, "A differential scheme for the effective moduli of composites," Mechanics of Materials, vol. 4, no. 1, pp. 1-16, 1985.

[33] S. Biwa, Y. Watanabe, S. Motogi, and N. Ohno, "Analysis of ultrasonic attenuation in particle-reinforced plastics by a differential scheme," Ultrasonics, vol. 43, no. 1, pp. 5-12, 2004.

[34] A. I. Beltzer and N. Brauner, "Acoustic waves in random discrete media via a differential scheme," Journal of Applied Physics, vol. 60, no. 2, pp. 538-540, 1986.

[35] K. G. Tay, S. L. Kek, and R. Abdul-Kahar, "A spreadsheet solution of a system of ordinary differential equations using the fourth-order Runge-Kutta method," Spreadsheets in Education, vol. 5, no. 2, pp. 1-10, 2012.

[36] R. McLaughlin, "A study of the differential scheme for composite materials," International Journal of Engineering Science, vol. 15, no. 4, pp. 237-244, 1977. 